\title{
Proceedings of the Association of British Neurologists and the British Neuropsychological Society, the Commonwealth Institute, London, 28-29 September 1995
}

\section{Platform presentations}

DIAGNOSIS OF THE NARCOLEPTIC SYNDROME JD Parkes, SY Chen, MJ Dahlitz. King's College School of Medicine and Dentistry and Institute of Psychiatry, London, UK

A clinical rating scale was developed and validated to aid the diagnosis of the narcoleptic syndrome. This scale determined sleep propensity with monotony, using the Epworth sleepiness scale, postural atonia after emotional excitement, and night sleep habits.

A total of 183 patients with both cataplexy and excessive daytime sleepiness were compared with 188 age and sex matched control subjects with normal sleep-wake habits. The propensity to excessive daytime sleepiness was five times greater in the narcoleptic group and propensity for postural atonia with laughter and other stimuli, 15 times greater. Sleep latency at night in narcoleptic patients was half that of controls. Motor parasomnias, leg kicking, sleep restlessness, sleep talking, and sleep paralysis were all more common in narcoleptic patients. Period to diagnosis of narcolepsy was over 10 years in one quarter of patients.

The best discriminant of the narcoleptic syndrome is cataplexy. This is essential for diagnosis. A characteristic feature is phasic circumoral muscle jerking. Normal subjects report greater muscle atonia with sudden noise than with laughter, vice versa in the narcoleptic syndrome. Additional features include excessive daytime sleepiness, presleep dream timing, short night sleep latency, insomnia, and frequent parasomnias.

[ABN]

SUDDEN DEATH IN EPILEPSY: AN OVERVIEW L Nashef, DR Fish, JWAS Sander, SD Shorvon. Institute of Neurology, London, UK

Sudden unexpected death in epilepsy is a well recognised occurrence. Incidence and possible mechanisms were investigated and overall conclusions based on different studies are reported.

Incidence of sudden death was high in cohorts with less well-controlled epilepsy, ranging between 1:200 and 1:300/year as compared with a reported incidence of $1: 1000 /$ year in a general population with epilepsy. An even higher incidence was found in those aged 15-34. Circumstantial evidence in favour of a seizure was found in most unwitnessed cases on detailed interview of self referred bereaved relatives.

In seizure recordings in patients undergoing EEG/video telemetry, apnoea ( $>10-63$ seconds) was seen in 20/47 seizures (10/17 patients). Central apnoea was seen in 10/10 patients, and obstructive apnoea in 3/10.
Transient bradycardia was seen in four patients and occurred with apnoea in $3 / 4$ suggesting the involvement of cardiorespiratory mechanisms.

Thus, most of these deaths are unwitnessed seizure deaths. Respiratory as well as cardiac mechanisms are likely to be involved. The potential for prevention needs to be considered in terms of more aggressive treatment of epilepsy and resuscitation in seizures.

[ABN]

LONG TERM PROGNOSIS OF EPILEPSY

J Stewart, E Hughes, AL Johnson, EH Reynolds. The Maudsley Hospital, London, UK

Prospective randomised comparative monotherapy trials have been previously reported for four antiepileptic drugs (phenobarbitone, phenytoin, carbamazepine, sodium valproate) in 243 adults and 167 children with newly diagnosed, previously untreated epilepsy - that is, two or more generalised tonic-clonic seizures or partial seizures with or without secondary generalisation within the previous 12 months. No difference in efficacy was found between the four drugs in adults, children, or in the combined groups. The most significant factors determining outcome at a median follow up of 36 months in the combined group were the number of seizures before commencing treatment, the interval between seizure onset and treatment, and the presence of neurolog$\mathrm{ical} /$ mental handicap.

The follow up of 130 adults and 167 children has now been extended for a median of 8.5 years and there were two and five year remission rates of $67 \%$ and $41 \%$ respectively in adults, and $77 \%$ and $41 \%$ respectively in children. Prognostic factors influencing two and five year remission rates have also been examined. Seizure number, seizure type, and neurological/mental handicap are the most important. Factors underlying late relapse of seizures and patient mortality have also been evaluated.

$[\mathrm{ABN}]$

ABSENCE STATUS IN ADULTS: A CLINICAL, EEG, VIDEO-EEG STUDY, AND FACTORS OF ERROR

S Giannakodimos, A Agathonikou, CP Panayiotopoulos. St Thomas' Hospital, London, UK

Absence status (AS) is a very interesting condition often unrecognised by the treating physicians, despite its importance in diagnosis and treatment. Twenty four adults over the age of 16 years with AS are presented. All had an extensive clinical study with EEG and/or video-EEG confirmation of absences.
Prevalence of AS was 5.9\% among 410 adults with epileptic disorders. In only five patients AS was recognised by the referring physicians and none of the patients was advised regarding treatment strategies and avoidance of the impending generalised tonic clonic seizures (GTCS).

The age at study was $42 \cdot 1(16 \cdot 3)$ years and 15 patients were female. The mean age at onset of AS was $30.0(14.4)$ years. All patients also had absences with a mean age at onset of $14.7(13.6)$ years and GTCS with a mean age at onset of $19 \cdot 2(12.5)$ years. All but three patients had idiopathic generalised epilepsies (IGEs): six the syndrome of phantom absences with GTCS, four perioral myoclonia with absences, two eyelid myoclonia with absences, two juvenile absence epilepsy, two juvenile myoclonic epilepsy, one predominantly photosensitive epilepsy, and four unclassified IGE. The mean minimum and maximum duration of AS ranged from 30 minutes to three days $(8.8(17 \cdot 1)$ hours) and from 30 minutes to 10 days $(31 \cdot 2(57 \cdot 3)$ hours) respectively. Symptoms varied from mild to severe cloudiness of consciousness, psychic-like manifestations but also with Kafka-like descriptions of experiential phenomena. Perioral or eyelid myoclonia, myoclonic jerks, and eyelid fluttering were consistent symptoms for those with the corresponding epileptic syndromes. Patients and friends or relatives and rarely the treating physicians were often aware of this condition which was invariably heralding a GTCS in 12 patients. Patients were often withdrawn to a "safe" place to have their "fit". Their descriptions were an invaluable tool in diagnosis and management. Even when patients were provided with a relevant medical report for the nearby accident and emergency department, treatment was delayed until a GTCS occurred to confirm the situation, which was not appreciated. $[\mathrm{ABN}]$

FUNCTIONAL ABNORMALITY IN CORTICAL DYSGENESIS

MP Richardson, MJ Koepp, DJ Brooks, DR Fish, JS Duncan. Hammersmith Hospital and Institute of Neurology, London, UK

Cortical dysgenesis is a cause of resistant focal epilepsy. Lesionectomy is often unsatisfactory, even in cases with well circumscribed lesions on MRI. This may be because MRI abnormalities represent the "tip of the iceberg"; the need exists for a technique which identifies the full extent of dysgenesis. Focal decreases in $\left[{ }^{11} \mathrm{C}\right]$ flumazenil binding have been previously identified in medial temporal sclerosis using PET, reflecting alterations in $\mathrm{GABA}_{\mathrm{A}}$ receptors. This technique has now been applied to cortical dysgenesis. $\left[{ }^{11} \mathrm{C}\right]$ flumazenil PET was 
performed in 14 patients with cortical dysgenesis and 24 normal controls. Using both a region of interest technique and statistical parametric mapping (which allows objective comparison between all voxels of each patient and the controls), regional abnormalities have been identified in [ $[$ C]flumazenil binding in 12 of the patients, using a statistical threshold of $\mathrm{P}<$ 0.001 . In 10 the functional abnormality was more extensive than the structural abnormality seen with MRI, and in eight patients there were multifocal abnormalities with receptor changes in regions with normal appearance on MRI. Lesions were frequently characterised by an increase in [" $\mathrm{C}]$ flumazenil binding rather than a decrease.

In conclusion, the technique of statistical parametric mapping allows detailed images of significant changes in regional ["C]flumazenil binding to be superimposed on to MRI and shows widespread areas of cortical dysfunction in patients with dysgenesis. These findings have implications in the pathogenesis of focal epilepsy and for the clinical management of such patients. $[\mathrm{ABN}]$

DIEVIILOPMINNT OF A DISTRICT STROKI: SERVICI:

SS Pollock. The Canterbury and Thanet Stroke Project, Kent, UK

The Canterbury and Thanet Stroke Project has used audit, communication, and publicity to justify the development of a comprehensive stroke policy in east Kent. The importance of reliable data was demonstrated through admissions surveys which showed admission rates up to $20 \%$ above the national average, inadequate therapy accounting for the low cost of $£ 3000$ per stroke admission, and inadequate information concerning the fate of the $£ 2.85$ million spent annually on stroke. Poor awareness of the available resources-for instance, less than $20 \%$ of general practitioners utilise the Stroke Information Centre-resulted in the introduction of the Stroke Discharge Brochure and regular multidisciplinary meetings.

The need for a neurovascular clinic was supported by the findings from a general practitioner questionnaire $(n=140)$. The numbers of transient ischaemic attacks thought to be seen annually were 10 times greater than predicted, and $40 \%$ of general practitioners found the diagnosis difficult. An audit of 34 young stroke admissions showed incomplete coding in half the cases and substantial omissions in management justifying the use of structured protocols. The presence of a stroke unit in one hospital shortened acute ward admissions by $20 \%$ ( $£ 400$ saved per patient) and attracted considerable non-NHS support which may have diverted attention from other aspects of care.

Increased integration of the service with social and voluntary agencies has resulted in joint funding for the family support worker and Stroke Information Centre and enhanced speech therapy through dysphasic support at less than $10 \%$ of the NHS cost.

Organisation of a policy that crosses hospital and community boundaries, social services, and the voluntary sector, embracing acute prevention, acute care, and rehabilitation is complex and requires the creation of the post of director of stroke services. [ABN]
RI:COVERY OF WORD RETRIEVAI AFTIER STROKE: FVIDENCE FRON PET STLDIIS E Warburton, RJS Wise, RSJ Frackowiak. Hammersmith Hospital, London, UK

The functional recovery of language after stroke is poorly understood. One hypothesis suggests that recovery is the result of partial lesioning-residual language skills being dependent on activity in the peri-infarct regions. An alternative hypothesis postulates a role for regions within the right hemisphere.

PET activation techniques have been used to investigate recovery in patients whose language abilities have improved after aphasic stroke and the results from two patients are reported to illustrate the findings. Both had improved sufficiently well to be able to perform a silent verb retrieval task in response to heard nouns while blood flow measurements were taking place. The PET images were coregistered with their MRI and compared with those of normal volunteers performing the same task

Verb retrieval activates a widely distributed network of language associated areas which include the left inferolateral temporal region, the left inferior parietal lobe, the dorsolateral prefrontal cortex (DLPFC), anterior cingulate, and the supplementary motor area (SMA). Activation of bilateral superior temporal cortices (STGs) in these tasks is dependent on the particular control state used.

The recovered aphasic patients activated the same network of regions (minus the infarcted areas) and foci of activation were demonstrated around the infarcted parts of the left temporal lobe, showing the importance of peri-infarct regions in the recovery of word retrieval. Novel areas of activation were found in the right prefrontal and left superior frontal regions, which may reflect the greater difficulty the patients had in performing the task. Additionally, there was a left-right difference in activation of the STG in these two patients demonstrating the ability of either temporal region to process the stimulus nouns

$[\mathrm{ABN}]$

RI:ORGANISATION OF THE EXECUTIVE MOTOR SYSTEM AFTER STROKE:

C Dettmers, KM Stephan, RN Lemon, RSJ Frackowiak. Hammersmith Hospital, London, UK

The aim of the study was to investigate reorganisation of the executive motor system after stroke, using PET. Regional cerebral blood (rCBF) was measured with oxygen-15 labelled water. Seven patients were scanned after recovery from different types of strokes. Twelve scans were repeated during rest and during repetitive key-press with the index finger of the affected hand with differen grades of force: $5 \%, 10 \%, 20 \%, 40 \%$, an $50 \%$ of the patient's maximal contraction (MVC).

Whereas the clinical symptoms at onset and the degree of recovery were similar in all patients, the individual patterns of activation were very different. Bilateral insular cortex, ventrolateral premotor cortex, second somatosensory cortex, and anterior operculum showed an altered $\mathrm{rCBF}$-force relation in individual patients indicating altered function and probably effective reorganisation. Group analysis suggested activation of the ipsilateral posterior supplementary motor area (pSMA) and the parietal cortex. M1 showed a binomial $\mathrm{rCBF}$-force relation with a sharp $\mathrm{rCBF}$ increase at $50 \%$ MVC in some patients. This indicates that patients recruit large numbers of motor neurons even at medium range force. This may explain why many patients with stroke experience increased sense of effort and fatigue. $[\mathrm{ABN}]$

A PROSPECTIVE CASI:-CONTROI. SILDY TO FXAMINE THE RFLATION BETWEE

CAMPYLOBACTER GLYLAI INHECTION AND GUILLAIN-BARRE SYNIOROMI

JH Rees, SE Soudain, NA Gregson, RAC Hughes. Guy's Hospital, London, UK

Although an association between Campylobacter jejuni (Cj) infection and Guillain-Barré syndrome (GBS) is known, its clinical, epidemiological, and immunological features have not been fully described. This association was further investigated with a prospective case-control study of patients admitted to hospitals in England and Wales between 1992 and 1994 Infection with $\mathrm{Cj}$ occurred in $26 \%$ of 103 patients compared with $2 \%$ of 94 household controls $(\mathrm{P}<0.001)$. Patients with $\mathrm{Cj}$ infection ( $\mathrm{Cj}$ positive) were more likely to have pure motor syndrome and to make a slower recovery due to the presence of axona degeneration. Furthermore, $\mathrm{Ci}$ positivity was an independent predictor of poor prognosis. There was a higher frequency of HLA DQB $1{ }^{\star} 03$ alleles $(\mathrm{P}=0.05)$ among $\mathrm{Cj}$ positive patients suggesting an immunogenetic predisposition to developing GBS after C infection. Antiganglioside $\mathrm{GM} 1$ antibodies (anti-GM1) occurred in $52 \%$ of the $\mathrm{Cj}$ positive patients compared with $16 \%$ of the $C$ negative patients $(P<0.001)$. The presence of anti-GM1 alone was not a poor prognostic indicator although patients with $\mathrm{Cj}$ and anti-GM1 had a worse outcome. These results suggest that $\mathrm{C}$ infection is a common event preceding GBS and that it is associated with a poor prognosis. The prognostic significance of anti-GM1 in $\mathrm{C}$-induced GBS is uncertain.

$[\mathrm{ABN}]$

RISSPIRATORY INSLYFICIINCY IN

NELRONOPATHIC DISORIDERS

( Hirsch, DH Miller, A Williams, GT Spencer. National Hospital and St Thomas' Hospital, London, UK

Twenty nine patients with a neuronopathic disorder were referred for assessment of respiratory insufficiency between 1978 and 994. Diagnoses included spinal muscular atrophy (six), chronic idiopathic demyelinat ing neuropathy (four), Vialetto-van Laere syndrome (three), hereditary motor and sensory neuropathy (three), and a miscellaneous group (five). Seven patients with Guillain-Barré syndrome (GBS) are also described who required long term ventilatory support for six months to seven year after the initial illness. Respiratory insufficiency occurred as a consequence of respira tory muscle weakness, impaired bulbar function, and restrictive lung defects. In some groups presentation was with progres sive nocturnal hypoventilation culminating in acute respiratory failure. Five patients with GBS or chronic idiopathic demyelinat ing neuropathy were weaned from ventila- 
tory support up to 18 months after the initial illness. The remaining 24 patients required continuous or nocturnal ventilatory support using intermittent positive pressure ventilation (13), negative pressure ventilation (four), nasal mask delivered intermitten positive pressure ventilation (four), nasal mask delivered continuous positive pressure ventilation (three), mouthpiece assisted ventilation by day (two) and rocking bed (one) None have been weaned from support after a period of ventilation ranging from one month to 10 years. Nine patients have subsequently died.

[ABN]

GENES FOR SPINAL MUSCULAR ATROPHY: PROBLEM SOLVED?

KE Morrison, KE Davies. John Radcliffe Hospital, Oxford, UK

In the mid-1980s, a positional cloning approach was commenced, with the aim of identifying the abnormal gene(s) in autosomal recessive childhood onset proximal spinal muscular atrophy (SMA). All three clinical forms were linked to chromosome $5 q 13$ markers in 1991 and subsequent genetic and physical mapping limited the disease gene to a region spanning less than $1000 \mathrm{~kb}$ of DNA. Isolation and analysis of candidate genes within this relatively small interval has proved immensely difficult. The region is highly unstable when subcloned into YAC and cosmid vectors and rich in chromosome 5 specific repetitive motifs. Genes and pseudogenes with complex duplication patterns abound.

Using a modified procedure for coincident sequence cloning, with cosmids containing microsatellites showing tight linkage disequilibrium with SMA, several gene sequences expressed in human brain which map within the region were isolated. These sequences lie very close to those of the survival motor neuron gene (SMN) and the neuronal apoptosis inhibitory protein gene (NAIP), both of which show deletions in some patients with SMA. All of these sequences are present in several copies, and no function has yet been assigned to any of the genes or the mutated copies. Carriers (phenotypically normal) with homozygous deletions for both SMN and NAIP have been identified, suggesting that additional genes nearby play a part in the SMA phenotype. SMA is clinically heterogeneous, both within and between families, and is more common in males: the genetic data thus far fail to explain these phenomena. [ABN]

ARTHROGRYPOSIS MULTIPLEX CONGENITA ASSOCIATED WITH AUTOANTIBODIES INHIBITING FETAL ACETYLCHOLINE RECEPTOR FUNCTION

A Vincent, S Riemersma, C Newland, D Beeson, SM Huson, J Newsom-Davis, D Hilton-Jones. John Radcliffe Hospital, Oxford, UK

Fetal arthrogryposis multiplex congenita (AMC) is characterised by multiple joint contractures, sometimes resulting in fetal or neonatal death, and associated with lack of movement in utero. AMC occurs occasionally with maternal myasthenia gravis (MG/AMC) suggesting a role for antiacetylcholine receptor (anti-AChR) antibodies. The fetal AChR comprises five subunits $\left(a_{2}\right.$, $\beta, \gamma, \delta$ ); the $\gamma$-subunit is gradually replaced by an $\varepsilon$-subunit, producing exclusively the adult form $\left(a_{2}, \beta, \varepsilon, \delta\right)$ by about 33 weeks. Serum antibodies have been recently reported that selectively inhibit fetal AChR function in a mother with an obstetric history of severe recurrent AMC who had never had MG. AMC associated with maternal MG might also be due to antibodies specific for fetal AChR.

Serum samples from four MG/AMC mothers were studied. Each had high titres of anti-AChR (50-1000 nM), and unusually high titres of antibodies inhibiting ${ }^{125} \mathrm{I}-a$ BuTx binding to human (fetal-type) AChR (50-150 $\mathrm{nM})$, indicating the presence of antibodies to the $\mathrm{ACh} / a$-BuTx binding sites. One MG/AMC serum has been tested in functional studies. It inhibited $\mathrm{AChR}$ function $>80 \%$ in Xenopus oocytes expressing fetal AChR, but had no effect on oocytes expressing adult $\mathrm{AChR}$

These results suggest that antibodies inhibiting fetal $\mathrm{AChR}$ function can cause AMC, and coexist with anti-(adult) $\mathrm{AChR}$ in mothers with MG/AMC.

[ABN]

SERUM ANTI-VOLTAGE GATED POTASSIUM CHANNEL ANTIBODIES DETECTED IN ACQUIRED NEUROMYOTONIA

IK Hart, C Waters, C Morris, D Beeson, A Vincent, O Pongs, J Newsom-Davis. John Radcliffe Infirmary, Oxford, UK, and Zentrum für Molekulare Neurobiologie, Universität Hamburg, Germany

Acquired neuromyotonia (NMT) is characterised by continuous muscle fibre activity due to peripheral nerve hyperexcitability. I has been previously shown that this may be caused by autoantibodies that interfere with neuronal ion channel function. An immunoprecipitation assay using ${ }^{125} \mathrm{I}-a$-dendrotoxin detects antibodies to voltage gated potassium channels (VGKCs) in only a small proportion of patients with NMT. A novel immunohistochemical approach has been developed. Serum samples were assayed by immunohistochemistry for IgG autoantibodies binding to recombinant human brain VGKCs or, as control, human muscle acetylcholine receptor (AChR) $a$-subunit proteins, expressed in Xenopus oocytes previously injected with the relevant cRNA. The results were confirmed in coded samples.

Eight of nine NMT serum samples bound to oocyte sections expressing HBK2. None bound to control sections expressing recombinant AChR $a$-subunit protein or to sections of water-injected oocytes. Two of two serum samples from patients with NMT, myasthenia gravis (MG), and anti-AChR antibodies bound to oocyte sections expressing either $\mathrm{HBK} 2$ or $\mathrm{AChR}$. Four of four serum samples from anti-AChR antibodypositive MG bound to oocyte sections expressing AChR $a$-subunit but not to sections expressing HBK2. None of 13 control serum samples bound oocytes injected with cRNA for $\mathrm{HBK} 2$ or $\mathrm{AChR}$, or with water.

These findings strongly suggest that antiVGKC antibodies are implicated in the pathogenesis of acquired NMT. They also indicate that this assay could be adapted to detect other autoantibodies for which the target gene has been cloned.

[ABN]
POSTPRANDIAL HYPOTENSION WORSENS THE PARKINSONIAN STATE IN PARKINSON'S

DISEASE

K Ray Chaudhuri, S Love-Jones, C Ellis, CJ Mathias, JD Parkes, PN Leigh. King's College School of Medicine and Dentistry and Institute of Psychiatry, London, UK

Patients with Parkinson's disease (PD) are often symptomatically worse after a meal and abnormal postprandial autonomic responses have been implicated. The postprandial autonomic responses and parkinsonian state in 20 patients with PD and 16 age matched controls were studied. Patients (mean age 65 (range 37-80) years, no autonomic failure) and controls (mean age 60 (34-74) years) were studied after an overnight fast before and after a standard balanced liquid meal $(570 \mathrm{kcal})$. Erect and supine blood pressure (BP), heart rate (HR), PD score (King's college and Hoehn and Yahr), muscle activity monitoring (wrist worn actimeter), blood glucose, insulin, and catecholamine concentrations were measured before and 15-60 minutes after the meal. Supine mean BP (mm Hg) fell significantly after a meal in patients with $\mathrm{PD}(\mathrm{P}<$ 0.05 ) with worsening of postural hypotension (greatest at 30-45 minutes, 94 (3) to 83 (3) $\mathrm{P}<0.05)$ but not in controls. Twelve patients had postural intolerance after the meal. The PD score worsened in all particularly at $30-45(6 \cdot 5-14)$ minutes, whereas muscle activity was reduced after the meal. Blood glucose and insulin rose after a meal in PD and controls. It is concluded that postprandial hypotension is common in PD and may indeed be responsible for worsening of the parkinsonian state after a meal. [ABN]

POSTURAL STABILITY IN PARKINSON'S DISEASE: EFFECT OF LEVODOPA

MJ Steiger, B Day, P Thompson, CD Marsden. Institute of Neurology, London UK

Levodopa preparations are said to be the most effective treatment in reversing the symptoms and signs of Parkinson's disease. The characteristics of postural sway were studied in 10 male patients with Parkinson's disease (mean age 49.1 (SD 7.3) years, range 37 to 58 years; mean disease duration $8 \cdot 5$ (SD 4.7) years, range 3 to 19 years), before and after a high dose levodopa challenge. Postural sway was measured using force platform (Kistler 9281B) to assess changes in foot torque. The position of the centre of foot pressure was calculated from the outputs of the force plate. Changes in body motion were measured using a noncontact, infrared motion detection system (Selspot II) that can assess movement of the body in three dimensions. Eight infrared emitting diodes were placed symmetrically on the left and right of the patient at the shoulders, hips, knees, and ankles.

The amplitude of fluctuations in velocity and position of body motion (averaged from eight sites), and of the centre of pressure in both the frontal and lateral planes were not significantly reduced after levodopa Although dopaminergic stimulation with levodopa reversed many of the clinical features of Parkinson's disease (particularly tremor and limb bradykinesia), it had no significant effect in reducing postural sway in the patients as a group. This suggests that 
the disorder of equilibrium in Parkinson's disease arises from additional non-dopaminergic responsive lesions. [ABN]

DIEPRISSSION IS A COMMON ANI) TRIEATABI I: CALSI: ()F PRESFNIIE I)EMENIIA

PN Cooper, J Ferran, K Wilson, E Ghadiali, $M$ Doran. Walton Centre, Liverpool, UK

The importance of a multidisciplinary approach to the investigation of presenile dementia has long been recognised, but uncertainty remains about whether it is possible to reliably discriminate between clinical presentations of different disorders. The records of 200 consecutive patients (mean age 53 years) referred to a presenile dementia service have been examined. The provisional initial diagnosis was reviewed after one year in the light of clinical follow up, the results of investigations including neuroimaging, and formal neuropsychological and psychiatric assessments. Standardised ICD-10 criteria were used to diagnose depression.

Twenty seven per cent were considered to have Alzheimer's disease, 18\% depressive pseudodementia, $16.5 \%$ vascular dementia, $29.5 \%$ had other specified disorders, and $8.5 \%$ remained unspecified. Depressive pseudodementia was poorly recognised by the referring doctor; and, moreover, less than one in six cases were identified at the initial clinical assessment. Provisional follow up results have suggested that these patients have responded well to appropriate treatment.

[ABN]

PRINARY PROGRISSIVI: APHASIA: TOWARDS A CIINICAI CLASSIFI(CATION

JR Hodges, $\mathrm{K}$ Patterson. University Neurology Unit and MRC Applied Psychology Unit, Cambridge, UK

Since Mesulam's seminal (1982) report, the term primary progressive aphasia (PPA) has been applied to patients with heterogeneous patterns of language deficit. Over the past five years 30 patients who fulfil strict criteria for PPA have been assessed, in detail. Three broad subgroups have emerged: (1) progressive fluent aphasia (semantic dementia), (2) progressive non-fluent aphasia, and (3) rapidly progressive aphasia with bulbar MND. The present paper concentrates on the first two subtypes and, in particular, contrasts five patients with semantic dementia and two with progressive non-fluent aphasia, all of whom have undergone longitudinal assessment using the same comprehensive neuropsychological test battery.

In semantic dementia, well articulated and syntactically correct but empty anomic speech is characteristic. The most prominent feature of the non-fluent syndrome is the severe speech distortion with phonological errors and agrammatic sentence structure. Performance on comprehension tasks separates the groups; the non-fluent patients show normal single-word comprehension, but considerable impairment on tests of syntactic comprehension, whereas those with semantic dementia demonstrate the opposite pattern. Performance on non-verbally mediated tests of semantic knowledge is impaired in semantic dementia only. The two forms of progressive aphasia have in common the sparing of perceptual and visuospatial skills, non-verbal problem solving abilities, and episodic memory.

Neuroradiological investigations (MRI) have shown selective and striking inferolateral left temporal lobe atrophy in all patients with semantic dementia: the changes in nonfluent cases involve left perisylvian structures more diffusely. [BNS]

LANGLA(IF DISORI)IR IN PROGRESSIVY SLPRANLCLLAR PALSY: A CONTROLLIEI) NELROPSYCHOLOGICAI STLDY

$\mathrm{T}$ Esmonde, $M$ Gibson, $\mathrm{E}$ Hoffner, J Hodges. University of Cambridge, Cambridge and Royal Victoria Hospital, Belfast, UK

Progressive supranuclear palsy (PSP) is known to cause cognitive decline which has been attributed to involvement of frontostriatal circuits. Disorders of language output have, however, not been well characterised. Based on recent observations, it was hypothesised that the disease may particularly affect this component of cognition. The results of a study of 25 patients with PSP and matched controls using an extensive neuropsychological battery are presented. Assessment of severity of disease was based on (1) duration of illness, (2) the degree of eye movement disorder, and (3) the Columbia rating scale (CRS). Patients with PSP showed significant deficits, compared with controls, in attentional, perceptual (visual and auditory), and semantic knowledge, and language tasks. Language output (fluency, discourse, and sentence completion) was severely reduced, with significantly more errors and failures of response. With the exception of attentional tasks, the degree of cognitive dysfunction did not correlate with the three clinical measures of disease severity. It is suggested that the language deficits may be due to a combination of a frontal dysexecutive syndrome and a breakdown in higher order linguistic planning, which seems to be a pervasive element of the disease even at an early stage.

$[\mathrm{ABN}]$

\section{PROGRISSSIVI: APHASIA:}

NEUROPSYCHOLO(GICAI, ANATOMICAL, ANI) HISTOLOOICAL RELATIONS

JS Snowden, HL Griffiths, D Neary, DMA

Mann. Manchester Royal Infirmary and Medical School, Manchester, UK

Slowly progressive aphasia was described by Mesulam in 1982 to refer to a non-fluent, language disorder, in which anomia, paraphasias, and agrammatical speech output occurred in the context of relatively preserved comprehension. It has become clear since then that circumscribed disorders of language resulting from degenerative brain disease may take a variety of forms, representing differential breakdown in psycholinguistic functions of phonology, morphology, syntax, and semantics. The clinicopathological relations between these diverse patterns of progressive aphasia have remained unresolved. The neuropsychological characteristics of eight patients are reported, whose clinical disorder was dominated by progressive language failure, and in whom necropsy examination of brain tissue has been carried out. In seven patients (three non-fluent, two fluent, and two mixed) a clinical diagnosis of non-Alzheimer frontotemporal cerebral degeneration had been made, and in one patient a diagnosis of focal Alzheimer's disease Neuropathological examination confirmed these clinical diagnoses. Neuropsychological differences between patients were mirrored by differences in distribution of atrophy within the frontal, temporal, and parietal lobes. The findings in patients with non-Alzheimer focal cerebral degeneration support the notion of a range of clinical presentations of a common underlying pathology.

[BNS]

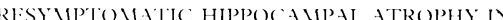
HAMHLLA AL /HLIMLR'S DISLASI

NC Fox, AM Kennedy, P Hartikainen, EK Warrington, JM Stevens, MN Rossor. The National Hospital for Neurology and Neurosurgery, London, UK

The hippocampal formation (HF) is known from pathological and MRI studies to be severely atrophied in established Alzheimer's disease (AD). Asymptomatic at risk members of a familial AD (FAD) pedigree with the APP 717 valine to glycine substitution within five years of the age at onset of the disease were recruited for longitudinal follow up; six members underwent serial MRI as well as neurological and neuropsychological assessments. Over a period of three years two subjects became clinically affected. Verbal and visual memory measures declined with the appearance of symptoms and in parallel with hippocampal loss. Volumetric measurement of the HF showed asymmetric atrophy to occur before the development of overt symptoms. The earliest structural changes in the hippocampal formation were shown to include the subiculum and CA1 areas; whereas atrophy was diffusely distributed along the length of the hippocampus, the posterior hippocampal head was particularly affected. Overall, a loss of up to $10 \%$ per year of the volume of the HF occurred in the two years over which symptoms first appeared. This study shows, that with this genetic mutation, detectable HF atrophy and memory deficits occur presymptomatically. This may have important implications for early diagnosis in AD more generally. [ABN]

AUDITORY RI:CO(BNITION DHFICITS IN NELROLOGICAL PATIFN" WITH BITLMPORAI I.ISIONS: A RI:PORT ON BRAIN MECHANISMS I:()R (OMPLASATION ANI) RI:COVERY

A Engelien, D Silbergsweig, E Stern, W Huber, O Frith, R Frackowiak. RWTH Aachen, Germany, Institute of Neurology, University College and Hammersmith Hospitals, London, UK, and Cornell University Medical Center, New York, USA

Auditory recognition deficits are rare and striking cognitive disorders. Several specific deficits have been described, ranging from cortical deafness to auditory agnosia and word deafness. Such individual case studies of human pathology have provided the most important evidence until the recent development of human in vivo functional brain imaging.

In this paper neuropsychological evidence 
is presented from two patients emphasising brain mechanisms of compensation for or recovery from central auditory deficits such as cortical deafness or complete auditory agnosia evolving into word deafness. These results are discussed in comparison with data on normative brain mechanisms of non-verbal sound categorisation (in a group of six normal volunteers). Both patients had two consecutive strokes, leading to bilateral perisylvian lesions touching the auditory cortices. Assessment by means of pure tone audiometry, acoustic reflexes, brain stem auditory evoked potentials, standardised intelligence tests, and the AAT (Aachen aphasia test) confirmed the diagnoses of specific central auditory disorders. PET activation studies described were performed with $\mathrm{H}_{2}{ }^{15} \mathrm{O}$ PET to measure regional cerebral blood flow (rCBF). A standardised statistical parametrical mapping (SPM(t)) protocol of image analysis and data processing was performed.

PET activation techniques demonstrated two possible brain mechanisms of compensation for or recovery from such deficits. In the first case with cortical deafness, volitional attentional activity enabled sound processing in spared cortical areas. In the second case with severe auditory agnosia bilateral recruitment of homologous regions (of a network normally subserving a certain cognitive task) is associated with the recovery of performance in this task. This seems to be the first demonstration of mechanisms of recovery from such specific auditory recognition deficits.

[BNS]

VERBAL AND NON-VERBAL MEMORY CORRELATES WITH HIPPOCAMPAL SUB-FIELD NEURON DENSITY

JM Oxbury, Z Matkovic, SM Oxbury, JH Morris, RW Hiorns, K Carpenter. Radcliffe Infirmary and University of Oxford, Oxford, UK

The relation between hippocampal subfields and human memory is poorly understood. Preoperative memory (verbal and non-verbal) and neuronal densities in hippocampal sub-fields CA1, CA2-3, CA4 and dentate fasciculus in temporal lobe specimens ( 33 right, 21 left) were examined from patients undergoing surgery for intractable epilepsy. Hippocampal sclerosis was the sole pathology and all patients achieved Engel I-II seizure outcome.

There were significant correlations between non-verbal memory (figure recall, Benton visual retention test and ReyOsterrieth) and the right CA4 neuronal density, and between verbal learning (WMS-R paired associate learning test trial 3 score) and the left CA4 neuronal density. The left CA4/verbal learning relation has been recognised previously but not the relation between the right CA4 and non-verbal memory.

Significant correlations were also found between delayed paragraph recall and neuronal densities in right CA1 and CA2-3, and between delayed reproduction of the Rey figure and left CA1 neuronal density.

The findings suggest that type specific memory (verbal, non-verbal) is mediated by CA4 neurons, and they support the view that the verbal $v$ non-verbal dichotomy may be less absolute than previously assumed. [ABN]
FUNCTIONAL AND ANATOMICAL BASIS OF CHINESE ACQUIRED DYSLEXIA

B Butterworth, W Yin, K Patterson, P Rudge. University College and National Hospital for Neurology and Neurosurgery, London and MRC Applied Psychology Unit, Cambridge, UK

Six cases of Chinese acquired dyslexia are described. Three cases seemed to correspond to the type "deep dyslexia" and three cases to "surface dyslexia". The patients with deep dyslexia made a high proportion of semantic errors and seemed to be unable to use sub-lexical information (phonetic radicals) to construct pronunciations. Unlike alphabetic cases, no effect of imageability was found. The patients with surface dyslexia, by contrast, made few semantic errors and relied on phonetic radicals too much, so that their pronunciations were analogous to regularisation errors in reading irregularly spelled English words. Unlike some alphabetic patients, they were not confused by homophones.

Anatomically, the deep and surface cases showed distinct patterns of damage, comparable with the reported damage of alphabetic patients although with greater subcortical involvement.

[BNS]

TRANSIENT EPILEPTIC AMNESIA: CLINICAL AND NEUROPSYCHOLOGICAL FEATURFS AZJ Zeman, SJ Boniface, JR Hodges. Addenbrooke's Hospital, Cambridge, UK

Although the possibility that focal seizures may present with episodes of transient amnesia was suggested more than a century ago, few definite cases of "transient epileptic amnesia" (TEA) have been documented.

Seven cases of TEA are described, diagnosed on the basis of their clinical features, EEG findings, and response to treatment. Mean age at onset was 64 (range 49-78) years, with a mean duration of attacks of three (range $0 \cdot 24-15$ ) years. Their only seizure type was TEA and all had experienced numerous attacks lasting from minutes to days. Retrograde amnesia was always a feature of the attacks but some could recall events during the attack. All had attacks in relation to sleep. Unequivocal temporal lobe epileptiform abnormalities were apparent in four of seven sleep EEGs; two had abnormal wake EEGs. These abnormalities were unilateral in one and bilateral in three cases. Four patients described interictal memory disturbance which affected particularly recall for salient personal events from recent years. Standard tests of verbal recall and recognition were, however, normal in all seven patients.

TEA is a distinctive manifestation of temporal lobe epilepsy and may occur as the predominant seizure type. Attacks are often brief, occur on waking, and sometimes permit recall of events during the ictus. The interictal memory disturbance may result from disruption of temporal lobe based long term memory consolidation.

[ABN]

EXECUTIVE DYSFUNCTION IN AMYOTROPHIC LATERAL SCLEROSIS

S Abrahams, LH Goldstein, A Al-Chalabi, CM Lloyd, RG Morris, A Pickering, DJ Brooks, PN Leigh. Institute of Psychiatry, St George's Hospital Medical School and Hammersmith Hospital, London, UK
Frontal lobe (executive) dysfunction in some patients with amyotrophic lateral sclerosis (ALS) has been consistently shown by neuropsychological tests of fluency and is supported by PET findings of dysfunction of the dorsolateral prefrontal cortex. Whether executive deficits are particularly associated with ALS accompanied by pseudobulbar palsy (upper motor neuron involvement in the cranial region) is evaluated.

Three groups of subjects were investigated: ALSpbp (pseudobulbar palsy) $\mathrm{n}=24 ; \quad$ ALSnon-pbp (non-pseudobulbar) $\mathrm{n}=28$; healthy controls, $\mathrm{n}=28$. Patients with ALS were impaired on a written verbal fluency test $(P<0.05)$, being most pronounced in the ALSpbp group ( $P<0.01$ ) On the random movement joystick test (involving the generation of random movement sequences with a four directional joystick) there was a strong tendency for the ALSpbp to be impaired $(P=0.06)$, being significantly poorer at generating random two-movement strings $(P<0.05)$. On the tower of Hanoi, a planning task, the ALSpbp group had shorter planning times on more complex trials $(P<0.05)$, suggesting impulsiveness. On a test of negative priming, measuring attention and inhibition, a deficit was found across patients with ALS $(\mathbf{P}=0.05)$

The data show executive dysfunction in ALS, which is more prominent in the pseudobulbar group. Such deficits are striking on tests of intrinsic generation, and support PET findings.

[BNS]

MEMORY RETRIEVAL: ATTEMPTING AND

SUCCEEDING-ROLE OF THE RIGHT FRONTAL LOBE

PC Fletcher, RJ Dolan, CD Frith, RSJ Frackowiak, MD Rugg. Hammersmith Hospital, London, UK

Previous functional imaging studies have implicated the right prefrontal cortex in memory retrieval. It is unclear, however, whether the activation of this region reflects retrieval effort or retrieval success.

Positron emission tomography was used to measure brain activity in six healthy male subjects during a word recognition memory task. Five minutes after a prescan study phase, subjects were presented with lists of words and required to identify "old" items (those seen in the study list: response "yes") and "new" items (not seen at study: response "no"). The degree of recognition was systematically varied during the scanning phase by manipulating the ratio of old:new words presented during this $50 \mathrm{sec}$ ond period. Across six scans, three ratios were each presented twice ( 0 old: 20 new; 4 old:16 new; 16 old:4 new). High performance on the tasks was ensured by employing a deep encoding task at study (in which subjects were required to use each word to generate a short sentence)

Alterations in brain activity due to response variation (differing ratios of "yes" and "no" responses) inherent in this set of conditions was controlled for using a simple target identification task in which the ratio of yes:no responses was varied analogously to that described above. Successful memory retrieval (correctly identifying old words), when compared with retrieval effort (correctly identifying new words, no old words being presented) was associated, predominantly, with right frontal activity. 
This result suggest that the right frontal activation at memory retrieval does not merely reflect the attempt to retrieve but is associated rather with the success of retrieval. $[\mathrm{ABN}]$

DUAL TASK PERFORMANCE IN FRONTAL PATIENTS WITH AND WITHOUT BEHAVIOURAL. IMPAIRMENT

S Della Sala, A Baddeley. University of Aberdeen, Aberdeen and MRC Applied Psychology Unit, Cambridge, UK

When patients with Alzheimer's disease (AD) are required to perform two tasks simultaneously, they are particularly impaired, even when pains are taken to ensure that the level of performance on the individual tasks is equated with that of age matched controls. A follow up longitudinal study showed that the disadvantage becomes more pronounced with the progress of the disease, whereas performance on the single task did not show this same degree of sensitivity. These data are consistent with the hypothesis of a deficit in $\mathrm{AD}$ of the central executive (CE) component of working memory.

The outcome of these studies seems to be a sound way of testing the central executive. However, the computerised version of the dual task paradigm employed in the series of studies reported above was impractical for everyday clinical use. Therefore, a paper and pencil version of the dual task was developed and validated, that could be more suitable for clinical practice. By means of this new version of the dual task, Baddeley's earlier findings with patients with $A D$ have been replicated.

Because it has been postulated that the locus of $\mathrm{CE}$ is within the frontal lobes a group of 24 patients with a lesion only in the prefrontal areas of the brain were tested. The patients were subdivided in two groups, those with $(n=12)$ and those without $(n=12)$ overt manifestation of behavioural impairments (for example, clinically defined dysexecutive syndrome). The dual task test proved to be a better predictor of the presence of a dysexecutive syndrome in these patients than either the Wisconsin test or verbal fluency, tests widely used as markers of frontal derangement. [BNS]

IMAGINED MOVEMENTS: ANATOMICAL BASIS RSJ Frackowiak, KM Stephan, C Dettmers. Institute of Neurology, London, UK

The organisation of the motor system in the human brain follows the principals of functional segregation and parallel outputs. Sportsmen and musicians often claim that practising movements in their mind improves performance. The neural substrate of preparation of set, imagined movements and executed movements was investigated.

Positron emission tomographic measurements of the distribution of cerebral blood flow, coregistered with anatomical images obtained with MRI were used. Preparation of set is associated with activity in dorsolateral prefrontal and anterior cingulate cortices. Imagined movements were associated with activity distributed throughout the lateral premotor, lateral inferior and medial premotor, supplementary motor, lateral and inferolateral parietal as well as insular cor- tices. Execution of movements additionally activated a nucleus of executive areas centred on the primary motor cortex, the caudal most parts of the premotor cortex, and the rostral most parts of the superior parietal cortex as well as the posterior half of the SMA and dorsal bank of anterior cingulate cortex.

This division of functions and nested hierarchal organisation may have implications for therapy and rehabilitation of motor disorders.

[ABN]

DIENCEPHALIC, TEMPORAL LOBE, AND FRONTAL AMNESIA: A NEUROPSYCHOLOGICAL AND PET STUDY

MD Kopelman, N Stanhope, P Marsden, D Lasserson. St Thomas' Hospital, London, UK

Many claims have been made for differences in the pattern of cognitive deficit between patients who have a diencephalic, temporal lobe, or frontal lobe lesion producing amnesia. These differences relate to such issues as recall $\mathrm{v}$ recognition memory, the response to semantic cueing, memory for spatial and temporal context, rates of forgetting, and the relative severity and temporal gradient (sparing of early memories) of retrograde amnesia. A study has recently been completed in 15 patients with diencephalic lesions, 14 patients with temporal lobe lesions, and 15 patients with frontal lobe lesions, who were selected on the basis of clinical, CT and/or EEG criteria. The patients then received cognitive testing, MRI imaging, and a "resting" FDG-PET scan as part of the research protocol. A brief account of some of the principal cognitive results, together with the main PET findings, were presented. [BNS]

HYPOTHERMIA AND MULTIPLE SCLEROSIS KD White, DJ Scoones, PK Newman. Middlesbrough General Hospital, Middlesbrough, UK

Spontaneous hypothermia in the absence of hypothyroidism is rare. The association with multiple sclerosis (MS) is an unusual and possibly under reported complication.

Five patients with clinically definite multiple sclerosis presented in the Northern Region over a period of three years, with acute relapses associated with hypothermia. Repeated episodes of hypothermia were seen in three. Thrombocytopaenia was associated with the hypothermia in four cases. Further investigation disclosed a tendency to chronic hypothermia and suggested an altered thermoregulatory set point in one case, where MRI, endocrine and autonomic studies failed to localise a lesion in the hypothalamus, but subsequent necropsy showed lesions in the posterior hypothalamic nucleus. This is the first case of multiple sclerosis and hypothermia in which hypothalamic lesions have been documented pathologically. In all five patients MS had been present for at least five years, four out of five were severely disabled (Kurzke score of $>6$ ), and all had clinical evidence of brainstem disturbance. Onset of hypothermia was insidious and associated with drowsiness, confusion, and snoring. We suggest that in such patients a predisposition to altered thermoregulation may occur due to direct involvement of the hypothalamus or from combined lesions affecting hypothalamic outflow to the brainstem and spinal cord.

[ABN]

NEURO-OTOLOGICAL FINDINGS IN

MIGRAINEURS WITH VERTIGO

PA Savundra, JD Carroll, RA Davies, LM Luxon. The National Hospital, London, UK

180 consecutive patients with vertigo (an illusion of movement) underwent neuro-otological examination. The prevalence of migraine was $27 \%$, higher than the prevalence in the normal population.

Of the 131 non-migraineurs, $96(73 \%)$ had only peripheral vestibulopathy (PV), of which $56(58 \%)$ were idiopathic, $25(19 \%)$ central vestibulopathy (CV), and $10(8 \%)$ no vestibulopathy, but in only one of these $(0 \cdot 76 \%)$, was the aetiology not identifiable. Of the 49 migraineurs, 10 were excluded because of coexistent disease with known vestibular sequelae. Of the remaining 39,12 (31\%) had migraine without aura, $27(69 \%)$ migraine with aura, including $17(42 \%)$ with a prodrome of vertigo. $10(26 \%)$ migraineurs had vertigo with no neuro-otological abnormality compared with only one non-migraineur ( $95 \%$ confidence interval $0 \cdot 11$ to $0 \cdot 39)$. Eight (30\%) migraineurs with aura and one (8\%) migraineur without aura exhibited CV (NS); 25 (64\%) of migraineurs exhibited PV, but there was no significant difference in prevalence in comparison with non-migraineurs with idiopathic PV (58.3\%)

Three conclusions emerge: (1) the high prevalence of vertigo with no neuro-otological abnormality in migraineurs suggests that there is transient vestibular dysfunction; (2) migraineurs with aura may be more likely to have $\mathrm{CV}$ than migraineurs without aura; (3) the prevalence of $\mathrm{PV}$ in migraineurs with vertigo is not in excess of non-migraineur with vertigo. [ABN]

BENIGN AND MOTOR NEURON DISEASE FASCICULATIONS ARE DIFFERENT: A MACROEMG STUDY

RJ Guiloff. Charing Cross Hospital, London, UK

Fasciculations (FUs) are frequent in motor neuron disease (MND) but may also be benign. 142 FUs and 130 voluntarily activated motor units (VUs) were recorded in the gastrocnemius $(n=6)$ and tibialis anterior ( $n=1$ ) muscles of seven consecutive patients with benign fasciculations (BF). 25 FUs had a macro EMG potential of similar shape, amplitude, and area to that of a VU (voluntarily activated FUs). The median macro EMG amplitudes $(P=0.05)$ and areas of the FU of the seven patients were higher than those of VUs; the mean number of spikes of the triggering single fibre potential (fibre density) was not significantly different in both types of unit. FUs that were activated voluntarily had similar macro EMG parameters to FUs that could not be so activated.

The findings differed from those of patients with MND $(n=10)$, in whom FUs had a higher fibre density $(P=0.024)$ but similar macro EMG parameters to VUs. The proportion of FUs that could be acti- 
vated voluntarily was higher in BF than in MND.

Benign fasciculation units seem to have a larger territory but similar peripheral microanatomy to the VUs whereas FUs in MND have a similar territory but a different peripheral microanatomy from VUs. The mechanisms of generation of abnormal discharges in BF may differ from those in MND. [ABN]

SUPEROXIDE DISMUTASE 1 (SOD1) AND MANGANESE DEPENDENT SOD (MNSOD) MRNA EXPRESSION IN VULNERABLE BRAIN REGIONS IN MOTOR NEURON DISEASE AND PARKINSON'S DISEASE

S Zeman, A Kingsbury, OJF Foster, PN Leigh. Institute of Psychiatry, London, UK

After identification of mutations in the antioxidant enzyme SOD1 in patients with familial motor neuron disease (MND), an investigation has been carried out on the activity of this and the related MnSOD in control and MND postmortem motor cortex and in Parkinson's disease (PD) substantia nigra (SN) by in situ hybridisation. Thus, expression of mRNA encoding these enzymes was localised and quantified within vulnerable cell groups.

A greater range of SOD1 mRNA expression was found in MND motor neurons than those of controls: levels were undetectable in $21.5 \%$ MND motor neurons (244 cells/five cases) compared with $6 \%$ of controls (338 cells/seven cases). Occasional MND motor neurons had unusually high SOD1 expression. Similarly, in melanised cells of the SN, SOD1 mRNA expression was undetectable in $15 \%$ control cells (202 cells/five cases) compared with $30.5 \%$ in PD (337 cells/eight cases), and again, some cells with unusually high expression were observed in cases of PD.

By contrast with biochemical studies MnSOD mRNA expression was dramatically decreased in melanised cells in PD $\mathrm{SN}-$ undetectable in $90 \%$ cells (90 cells/seven cases), compared with $59 \%$ in controls (120 cells/five cases). Conversely, a trend towards increased MnSOD mRNA expression in MND motor neurons was seen.

These data demonstrate alterations in antioxidant mechanisms in affected cell groups in MND and PD which may determine their vulnerability to the degenerative process.

[ABN]

ELEMENTARY VISUAL HALLUCINATIONS IN MIGRAINE AND EPILEPSY

CP Panayiotopoulos. St Thomas' Hospital, London, UK

This is a prospective study to compare the elementary visual hallucinations of 50 patients with migraine and 24 patients with occipital lobe seizures.

In migraine, elementary visual hallucinations were predominantly black and white with linear and zigzag patterns. The zigzag shaped lights were commonly seen in arcuate shapes across homonymous visual fields. Rarely, patients described circular lights or rounded obscuration of vision but the linear patterns of the fortification spectra were dominating. Colours were often described but they were not predominant, mainly occurring in the periphery of the visual hallucinations or appearing as faint coloured lines along with the zigzag bright streaks or flashes of light.

In occipital lobe epilepsy, hallucinations were predominantly multicoloured, with circular or spherical patterns. Predominantly square or triangular multicoloured patterns were described by three patients and in another one seizures would start with a monocoloured ball of bright red. None of the patients described visual hallucinations similar to the scintillating scotoma, photopsias, fortification spectra, or teichopsias of migraine although such terms were erroneously used in medical records and some of them were erroneously diagnosed as migraine or epileptic seizures triggered by migraine. Claims that epileptic seizures are triggered by migraine may reflect diagnostic problems. No such a case was ever recorded in over 6000 patients with epileptic disorders seen by this author.

[ABN]

SPEECH DISORDER IN ALZHEIMER'S DISEASE AJ Astell, TA Harley. University of Warwick, Coventry, UK

Explanations of the speech disorder in probable Alzheimer's disease (PRAD) predominantly focus on underlying semantic disturbance. The cause of this is either an impairment of access to intact representations or actual impairment of the representations. A two stage model of lexicalisation in speech production was used to examine this disorder. The two stage model has separate levels of representation for semantic and lexical items enabling discrimination between problems with the accessing and storage of concept knowledge and problems with the labels for such concepts. The response consistency to a battery of tasks designed to distinguish between competing hypotheses derived from the two stage model was explored. The results suggest that the problem lies in accessing lexical items. [BNS]

SEMANTIC IMPAIRMENT WITHOUT SURFACE DYSLEXIA: UNSTICKING THE SEMANTIC GLUE HYPOTHESIS

MA Lambon Ralph, AW Ellis, S Franklin. University of York, UK

Recent models of reading, including some connectionist simulations, have suggested that the process of reading aloud is reliant, at least in part, on the support of intact semantic representations (the "semantic glue hypothesis"). Without the assistance of the semantic system, these models become a surface dyslexic: a reading disorder in which the reader has specific problems in translating orthography to phonology of low frequency, irregularly spelt words. This apparently causal link between semantics and correct reading has been demonstrated in some patients with progressive brain disorders including dementia, Alzheimer type (DAT) and some dementias which show a more circumscribed area of atrophy.

A patient with DAT is presented, who, despite poor semantics, shows extremely good reading ability even for low frequency, irregular words. This strong dissociation is discussed in relation to the semantic glue hypothesis and traditional dual route models of reading. The data are also used to highlight the advantages and disadvantages of using associations and dissociations as methodological tools within neuropsychological research.

[BNS]

A NEUROPSYCHOLOGICAL AND

PSYCHOPHARMACOLOGICAL MODEL OF POOR MOTIVATION AFTER BRAIN INJURY

J Powell, $S$ al-Adawi, $\mathbf{R}$ Greenwood. University of London, Institute of Psychiatry and Homerton Hospital, London, UK

Patients with single incident brain damage often present with profound disorders of motivation, manifest in both therapy and daily life as a generalised passivity and failure to initiate even simple behaviours. A possible explanation of this is that their brain injury has involved damage to the brain circuitry underlying the ability to respond to sources of potential reward. The particular system implicated is the mesolimbic dopamine circuitry, which originates in the nucleus accumbens and projects in a reciprocal loop to the frontal cortex.

This model would predict close relations between motivational impairment eviden observationally, reduced sensitivity to normal sources of reward, and cognitive deficits of a frontal lobe type. The present study investigated these interrelations in a consecutive series of 50 patients admitted to the neurological rehabilitation unit at Homerton Hospital, having sustained either traumatic brain injury or subarachnoid haemorrhage. Structured therapist ratings of motivation over several treatment sessions were recorded, and an objective experimental test of responsiveness to incentive (the CAR ROT) was devised. This test was administered in a formal test setting along with an extensive range of cognitive tests of reasoning ability, memory, and frontal lobe function. Measures of affective state were also taken.

Highly significant correlations were found between motivation, reward sensitivity, and frontal lobe function as predicted. Factor analysis corroborated the close relation between these variables and a clear separation from measures of general intellectual ability and mood. In a related study, treatment of 10 consecutive patients with a dopamine agonist (bromocriptine) was associated with parallel improvements in all these variables in all 10 patients. [BNS]

CO-OCCURRENCE OF DYNAMIC APHASIA AND PHONOLOGICAL DYSLEXIA IN PRIMARY PROGRESSIVE APHASIA

S Tedman, JJ Downes, $M$ Doran, JR Hanley, E Ghadiali. University of Liverpool, and The Walton Centre for Neurology and Neurosurgery, Liverpool, UK

Primary progressive aphasia (PPA) is a heterogeneous syndrome believed to be characterised by a gradual dissolution of language with relative preservation of episodic/autobiographical memory and other cognitive abilities. According to Mesulam and Weintraub, the initial objective evidence for PPA takes the form of anomic difficulties with phonemic, rather than semantic, paraphasias, which progress to more severe fluent or nonfluent aphasias. Hodges has suggested that 
the "fluent" variety be designated semantic dementia and this has been most thoroughly investigated by the Cambridge group. In this paper a patient is described, who for three years has shown a progressive severe dysfluency such that conversational output is now mostly restricted to single words. As with PPA this language impairment is not accompanied by other significant cognitive impairments-episodic memory and visuoperceptual functions are normal. Unlike the classic PPA picture, however, his confrontation naming ability is within normal limits. He shows a deficit in the processing of sentences and in this respect resembles the PPA patient with "dynamic aphasia" described by Kartsounis. Additionally, however, detailed investigation of single word processing demonstrated normal reading and repetition of both regular and irregular words but a complete inability to assign the correct phonology to non-words, a pattern consistent with phonological dyslexia. [BNS]

NEUROPSYCHOLOGICAL FUNCTIONING IN A GROUP OF PATIENTS WITH ANOXIC BRAIN DAMAGE

BA Wilson. MRC Applied Psychology Unit, Cambridge, UK

This study describes the neuropsychological status of 14 people with anoxic brain damage referred for assessment. Six had attempted suicide with carbon monoxide poisoning, four had a myocardial infarction followed by cerebral anoxia, three had sustained an anaesthetic accident, and one had almost drowned.

The sample comprised 11 men and three women ranging in age from 16 to 55 years.

Six of the patients were followed up five to 10 years later. The three young men with the pure amnesic syndrome (two from $\mathrm{CO}$ poisoning, one from drowning) had done reasonably well, one was in paid employment, had married since the anoxic episode, and was hoping to start a family. The othe two were attending college. All three had shown some improvement on standardised memory tests but were still memory impaired.

One man with memory and frontal lobe problems, who was 47 years old at the time of his suicide attempt, was in long term psychiatric care. Another, who had attempted suicide at the age of 29 and who had been left with very severe intellectual handicaps was living in his own home with a rota of volunteer helpers. Despite some improvement he still had an apperceptive agnosia. The final patient was a young woman who had sustained massive brain damage as a result of an anaesthetic accident. She was living at home with relatives and attending a day centre two days a week. Again some improvement could be seen on the neuropsychological assessment but she remained extremely handicapped.

[BNS]

\section{Poster presentations}

PURE ALEXIA IN CHINESE

W Yin, B Butterworth. University College, London, UK
This poster reports the first detailed study of Chinese pure alexia. Two patients were investigated. One patient had circumscribed damage in the left occipital lobe in the same location as the classic cases of alphabetic pure alexia. In the second case, this area was involved along with some others. This similarity with alphabetic pure alexia undermines suggestions that Chinese character have a different localisation from alphabetic words.

Both patients presented with a right hemianopia and both were also found to show a "radical by radical" reading strategy, which is analogous-and possibly functionally equivalent-to "letter by letter" reading in alphabetic patients.

Experiments showed evidence of impairment in the word form system. Both patients had difficulty identifying real words, but were able reliably to reject non-words. It is proposed that the lexicon of orthographic word forms has been depleted by damage, leaving the more common characters that also act as radicals. This account explains poor reading aloud and the pattern of lexical decision performance, and also radical by radical reading. [BNS]

${ }^{1}$ C-FLUMAZENIL PET STUDIES IN MOTOR NEURON DISEASE

PN Leigh, CM Lloyd, S Abrahams, DB Brooks. Institute of Psychiatry, King's College School of Medicine and Dentistry, and Hammersmith Hospital, London, UK

${ }^{11} \mathrm{C}$-flumazenil was used as a ligand for PET to study the extent of cortical neuronal damage in motor neuron disease (MND).

Patients with clinically probable or definite $\mathrm{MND}$, according to the El Escorial criteria, and age and sex matched controls were selected. PET was performed after an intravenous bolus of ${ }^{11} \mathrm{C}$-flumazenil, a ligand which binds to GABAa receptors. Flumazenil binding was calculated as regional volumes of distribution (Vds). Statistical parametric maps (SPMs) were generated comparing the two groups, with a significance level set at $0 \cdot 01$.

Significantly reduced ${ }^{11} \mathrm{C}$-flumazenil binding was detected in the motor cortex and the dorsolateral and medial prefrontal regions in the patients with $M N D$ compared with the controls.

This study shows that ${ }^{11} \mathrm{C}$-flumazenil binding discloses evidence of loss of GABAa receptor sites in the prefrontal cortex of nondemented patients with typical MND; and it is concluded that this reflects loss of neurons bearing GABAa receptors. This supports previous PET activation studies, using regional blood flow. It is suggested that neuronal loss in the prefrontal region may be a consistent feature in the pathology of MND. Changes in "C-flumazenil binding may provide an objective means of monitoring the progression of cortical degeneration.

[ABN]

IDENTIFICATION AND TESTING OF INTRACRANIAL COLLATERALS USING TRANSCRANIAL DOPPLER AND COLOUR CODED IMAGING

PJ Martin, AR Naylor. The Walton Centre, Liverpool and Leicester Royal Infirmary, Leicester, UK
Necropsy studies suggest that in up to $80 \%$ of people the circle of Willis is incomplete. The presence of primary intracrania Willisian) collaterals should protect agains ipsilateral cerebral ischaemia during occlusion of the extracranial internal carotid artery (ICA).

Transcranial colour coded sonography (TCCS) was used to assess primary collaterals in 100 patients with ICA stenosis. Their functional importance was determined in 76 patients during carotid endaraterectomy by transcranial Doppler measurement of blood flow velocity in the ipsilateral middle cerebral artery (MCAV) before and after carotid clamping. All patients had unilateral ICA disease of $>60 \%$. Thirty four ICAs $(17 \%)$ were occluded, 70 vessels $(35 \%)$ were stenosed by $80-99 \%, 43$ vessels $(21.5 \%)$ were stenosed by $60-79 \%$, and 53 ICAs had stenosis of $<60 \%$. Temporal hyperostosis precluded TCCS in 15 patients (15\%). Anterior cerebral/communicating artery and posterior cerebral/communicating artery collaterals were detected in 40 patients $(49 \%)$ and 22 patients $(27 \%)$ respectively. No patients with ICA disease of $<80 \%$ had established collaterals. Patients with established Willisian collaterals showed higher postclamp MCAV as a proportion of the preclamp value during CEA (72 (62-81)\%; median $(95 \% \mathrm{CI})$ ) than those without primary collaterals $(46 \%(34-58) ; P=0.02)$

TCCS allows non-invasive assessment of intracranial primary collaterals, the functional importance of which is recognised during abrupt haemodynamic challenge. It may determine which patients are at greatest risk of ischaemia during cerebral revascularisation.

$[\mathrm{ABN}]$

ASSESSMENT OF THE VALUE OF NOCTURNAL POLYSOMNOGRAPHY IN THE INITIAL

INVESTIGATION OF SUSPECTED OBSTRUCTIVE SLEEP APNOEA

A Williams, $\mathbf{R}$ Howard. St Thomas' Hospital, London, UK

Nocturnal polysomnography (NPSG) is recommended in the investigation of suspected obstructive sleep apnoea (OSA) but it is expensive and of limited availability. To determine the diagnostic value of NPSG 97 consecutive patients referred for evaluation of possible OSA were reviewed.

NPSG was used to establish the diagnosis of OSA as well as (1) to confirm sleep and sleep states, (2) to determine sleep efficiency, and (3) to diagnose other sleep disorders. Oximetry was separately assessed by visual analysis for a pattern of desaturations consistent with OSA.

Seventy seven patients had OSA (respiratory disturbance index $37(28)$ ). Fifteen of these patients with OSA had another diagnosis (mainly periodic limb movement disorder, (PLM)) and seven of the remaining 20 patients without OSA had another possibly significant diagnosis (mainly PLM or early REM). Sufficient sleep was observed in all but five and REM was entered in all but two of the 97. Fifty three of those with polysomnographically confirmed OSA (75\%) were correctly identified from oximetry traces with $>4 \%$ dips and an additional $15(19 \%$; total $94 \%)$ correctly identified with $2-3 \%$ dips.

It is concluded that although full NPSG may not be necessary in the initial evaluation 
of suspected OSA, oximetry as currently interpreted is insufficient to fully evaluate such patients and a modified visual analysis of the oximetry trace together with a record of limb movements is suggested. [ABN]

ANTIGLYCOLIPID ANTIBODIES IN PATIENTS WITH RHEUMATOID ARTHRITIS COMPLICATED BY PERIPHERAL NEUROPATHY AM Salih, NB Nixon, P Heath, CP Hawkins, PT Dawes, D Mattey. North Staffordshire Royal Infirmary, Stoke on Trent, UK

Humoral immunological mechanisms might be responsible for the development of peripheral neuropathy in rheumatoid arthritis (RA). Antibodies against acidic glycolipid molecules are found in several autoimmune peripheral neuropathies (PN). The objectives of this study were (1) to relate clinical features of RA with $\mathrm{PN}$; (2) to investigate the presence of antiglycolipid antibodies in patients with rheumatoid arthritis complicated by clinical neuropathy.

Twenty eight patients had their RA disease assessed and their PN quantified by the neurologic disability score (NDS) and the neuropathy sensory symptom score. Thirty eight patients with RA without PN were also assessed and 28 healthy volunteers were recruited. Serum samples from all patients and controls were tested for the presence of IgG and IgM anti-GM1 and sulphatide antibodies by ELISA. Values more than 2SD above the mean for the RA controls are considered abnormal.

There were more males among the patients with RA with PN and those patients had more extraarticular features $(P<0.05)$, and higher erosive scores $(P<0.001)$. Clinical sensorimotor neuropathy was found among $23(82 \%)$ patients and pure motor and sensory neuropathy was found in two $(7 \%)$ and three $(11 \%)$ patients respectively. Twelve patients with RA with PN (43\%) and two RA controls (5\%) had positive titres against one or more glycolipids $(P<0.001)$. The NDS correlated with RA duration $(P<0.05)$, and with the presence of IgM GM1 $(P<0.01)$ and $\operatorname{IgM}$ sulphatide $(P<0.05)$ antibodies.

It is concluded that $\mathrm{PN}$ is associated with more severe RA disease and a significant proportion of these patients had antiglycolipid antibodies. Sensorimotor neuropathy is the commonest subset among patients with RA.

$[\mathrm{ABN}]$

AUDIT OF HOSPITAL CODING AND

INVESTIGATION OF PATIENTS WITH STROKE UNDER 65

P Young, SS Pollock. The Canterbury and Thanet Stroke Project, Kent, UK

Management of young patients with stroke is often unsatisfactory. As part of its aim to develop a financially viable stroke policy in east Kent the Canterbury and Thanet Stroke Project has undertaken a joint retrospective audit of acute stroke admissions under 65.

The investigation and coding of all patients with acute stroke under 65 admitted in the six months to March 1992 were audited by examination of the medical records.

Thirty four patients were identified. In half, the diagnosis was correctly coded and in 11 the separation between acute stroke, haemorrhage, and infarction was inappropriate. In five patients no acute stroke code was used. Nine patients died, 15 were discharged home, and 10 more discharged to other wards, with only four going to the stroke unit. Clerking or previous notes disclosed risk factors in all patients (total 100) 22 having three or more. However, investigation of patients $(n=30)$ was incomplete and led to identification of only 10 more risk factors.

In 12 patients the erythrocyte sedimentation rate was omitted and in nine the random glucose. Only seven patients had fasting lipids checked and in a quarter no ECG was undertaken. CT was performed in 17 instances. Of the 139 tests performed 36 were significantly abnormal including three of five ECGs.

Fifteen of the 25 survivors received physiotherapy and 10 speech therapy. A quarter saw an occupational therapist and only two a social worker.

The incomplete investigation and diagnosis demonstrated here suggests that management of this group of patients could be improved. The findings justify the introduction of structured protocols in stroke management. [ABN]

COMPUTER PROGRAM FOR PARKINSON'S DISEASE

AJ Fowle, CG Clough. Kings College Hospital, London, UK

Neurobase PD is a program for use with patients with Parkinson's disease. It supports management of individual patients and also provides a basis for research and audit. In the community, it can be used by the Parkinson's disease nurse on a portable computer and will support a multidisciplinary approach. It can be used to follow the same patients through both district general hospital and regional specialist clinics. In hospital use it runs across a network and can be integrated with a central PAS system.

The core clinical material includes a structured medical history and the following assessment scales: Barthel ADL index; united Parkinson's disease rating scale; Columbian rating scale; Fahn-Marsden dystonia rating scale. Further scales can be added to the program. Diagnoses are recorded with ICD 10 codes. The interface is designed for use by clinical staff, who need have little knowledge of computers. All coding is carried out by the program without the need for paper reference documents. An extensive on line help system is built into the program.

The program is written in Paradox for Windows, which provides security for the data as well as powerful tools for analysis. [ABN]

SIGNAL INTENSITY ON MR IMAGES OF THE BASAL GANGLIA IN MULTIPLE SCLEROSIS J Grimaud, J Millar, JW Thorpe, IF Moseley, WI McDonald, DH Miller. Institute of Neurology, London, UK
It has been reported that a relative reduction in signal intensity on T2 weighted MRI may be seen in the basal ganglia of patients with multiple sclerosis and furthermore that this is due to excessive iron deposition. However, the basal ganglia are rarely involved clinically or pathologically in multiple sclerosis, casting some doubt on this finding. Studies with MRI were therefore carried out in 46 patients with definite multiple sclerosis and 42 age matched controls. Contiguous, $5 \mathrm{~mm}$ thick axial dual echo spin echo images of the brain were obtained on a 1.5T MR imager. Visual rating scales were used to measure the lesion load as well as the signal intensity of the globus pallidus, putamen, caudate nucleus, substantia nigra, red nucleus, and thalamus. There was a mild degree of hypointensity in the patient group in the thalamus only. Hypointensity of the thalamus and putamen never exceeded that of the globus pallidus.

Basal ganglia hypointensity is rarely, if ever, found in multiple sclerosis and is not a useful radiological sign.

[ABN]

A PILOT INVESTIGATION OF PARKINSON'S DISEASE IN AN AFRO-CARIBBEAN MIGRANT COMMUNITY IN LONDON

M Richards, K Ray Chaudhuri. Institute of Psychiatry, London, UK

Several studies have reported a low prevalence of Parkinson's disease (PD) in populations of African origin. It is unclear, however, whether these populations are protected against PD or whether they are inadequately represented in these studies. As a preliminary investigation of this question, a pilot study of PD was undertaken among older AfroCaribbean migrants to the United Kingdom To establish a representative sample, a community register of Afro-Caribbean and white people aged 65 and older was compiled by door knocking within an electoral ward in the Southwark borough of Greater London. Forty five Afro-Caribbean and 45 age and sex matched white subjects from this register completed a structured interview for medical history. No white subject had been diagnosed with PD. However, three male AfroCaribbean subjects had a diagnosis of PD confirmed by a senior neurologist. These subjects also reported a history of cardiovascular disease, with past stroke in two subjects and treated hypertension in all three. This pilot study therefore offers no support to the suggestion that Afro-Caribbean migrants are protected against $P D$. $[\mathrm{ABN}]$

MANAGEMENT OF NON-TRAUMATIC SPINAL CORD INJURY

V Stevenson, ED Playford, DW Langdon, AJ Thompson. Institute of Neurology, London, UK

The management of acute traumatic spinal cord injury is well established. Experience of non-traumatic cord injury which has varying causes and uncertain outcome is more limited.

The neurorehabilitation unit at the National Hospital for Neurology and Neurosurgery has managed 49 such patients with non-traumatic cord injury in the past two years. These comprise 20 patients with cervical myelopathy secondary to cervical spondy- 
losis, most of whom had recently undergone surgical decompression; 15 patients with intrinsic cord abnormalities including syrinxes, seven patients with inflammatory disease including hereditary spastic paraparesis and tropical spastic paraparesis, and seven patients with spinal cord infarcts.

The age ranged from 17-88 (mean 54) years. Mean duration of stay was 40 days and did not differ significantly between each disease group. Under half of the patients were considered to have made some neurological improvement, and all but one improved when assessed using disability scales. This functional improvement did not correlate with the patients' age, initial disability, or level of the lesion, but was related to the length of stay in the unit. It was greatest in those with neurological recovery, and least in those with progressive diseases.

Rehabilitation of spinal cord lesions seems to be effective; outcome is influenced by the underlying disease and the duration of rehabilitation.

$[\mathrm{ABN}]$

BLINK REFLEX IN THE ASSESSMENT OF BRAINSTEM FUNCTION IN MITOCHONDRIAL MYOPATHIES

M Koutroumanidis, A Papadimitriou, E Bouzas, T Avramidis, $\mathbf{P}$ Papathanassopoulos, R Howard, T Papapetropoulos. S Thomas' Hospital, London, UK, Red Cross Hospital, Athens, and University of Patras, Greece

To investigate the brainstem function in mitochondrial myopathies (MMs), systematic blink reflex (BR) studies were performed in 17 patients with histochemically and genetically confirmed MM. Two of these patients had MERRF syndrome, one Kearns-Sayre syndrome (KSS) and 14 chronic progressive external ophthalmoplegia (CPEO) without symptoms and signs of CNS involvement. R1 latency was normal in all cases. R2 latency was abnormal in the two patients with MERRF and in the one with KSS. Statistical analysis, however, showed a significant delay of R1 and R2 at 0.05 and $0.001 \%$ respectively. The relative amplitudes were also invariably suppressed. The $M$ response of the orbicularis oculi muscle was of normal latency and amplitude and EMG examination disclosed subtle myopathic changes. No evidence of subclinical peripheral neuropathy was detected. Al abnormalities displayed a fairly symmetric distribution. These findings argue for a diffuse "central" dysfunction at the brainstem level, rather than a distinct segmental lesion. The discrepancy between polysynaptic R2 and oligosynaptic R1 indicates a greater dysfacilitation at the level of interneurons than at the motor neuron which serves as the final common path. The underlying mechanism presumably involves a, still clinically silent, cerebral cellular metabolic impairmen caused by the biochemical defects of the mitochondrial respiratory chain. These findings suggest that the BR may be useful in MMs for, beyond its clinical value, it may document CNS pathophysiology. [ABN]

SOUTH LONDON COMMUNITY STROKE REGISTER: OBJECTIVES AND METHODOLOGY JA Stewart, RS Howard, R Ross-Russell, AY Rudd, CDA Wolfe. St Thomas' Hospital, London, UK
The aims are to determine the incidence and natural history of the subtypes of stroke, the resource use, and the cost of stroke care. The setting is a multiethnic, nner city population of 234150

Patients of all ages with first ever stroke occurring after 1 January 1995 are registered. Using "hot pursuit" techniques, the incidence of stroke by ethnic group and stroke subtype will be determined. Standardised data collection questionnaires capture information on patients' prestroke functional status, demography, risk factors, stroke type and severity, and use of health service resources.

Patients are followed up at three months, one year, and yearly thereafter up to five years. Each patient is examined by one of the study physicians. Standard scales are used to determine patient impairment, disability, handicap, and quality of life. These include the mini-mental state examination and the motricity index, Barthel ADL, the Frenchay activities index, and the modified SF-36. Patient and carer satisfaction, and caregiver strain index are used to assess the quality of care.

These data will increase knowledge of the natural history of the subtypes of stroke and allow assessment of the long term needs of stroke survivors. The relation between resource use, cost, and outcome of stroke will be examined and these data will help to identify those aspects of care which improve outcome, so that more efficient, cost effective care strategies can be developed. [ABN]

IMMUNOLOGICAL CROSS REACTIVITY BETWEEN CAMPYLOBACTER YEFUNI ANTIGENS AND ANTIGANGLIOSIDE GMI ANTIBODIES IN GUILLAIN-BARRÉ SYNDROME

JH Rees, NA Gregson, RAC Hughes. Guy's Hospital, London, UK

Campylobacter jejuni (Cj) infection is a common antecedent event in GuillainBarré syndrome (GBS) although the mechanisms underlining this association are not known. About $50 \%$ of patients with $\mathrm{Cj}$ induced GBS have antibodies to ganglioside GM1 (anti-GM1). Purified IgG anti-GM1 from $\mathrm{Cj}$ positive patients with $\mathrm{GBS}$ reacted with lipopolysaccharide (LPS) from certain patients' organisms but not with any proteins. There was no correlation between the pattern of LPS reactivity and the clinical or electrophysiological features of the individual patient's disease. However, the predominant IgG subclass of anti-LPS antibodies in patients with pure axonal neuropathies was IgG1 suggesting the involvement of $\mathrm{T}$ cells in the generation of anti-carbohydrate responses in this group of patients. This finding suggests that a peptide antigen may be involved in the presentation of GM1 gangliose epitopes to $T$ cells. Although the nature of this peptide antigen is not known, a potential candidate might be $\mathrm{Cj}$ enterotoxin which binds with high affinity to ganglioside GM1.

[ABN]

TWIN COLLECTION IN PARKINSON'S DISEASE USING A NEW METHOD

CH Hawkes, NJ Phillips, CD Marsden, AVH Schapira, A Macdonald. Ipswich Hospital, Ipswich and Institute of Neurology, London, UK
Despite many family and twin studies, the inheritance of Parkinson's disease (PD) is unknown, except for a minority of families with dominant inheritance. A new method of twin collection has been piloted to overcome the problems of sample size and bias that have compromised earlier studies.

About $70 \%$ of general practitioners have computerised records. East Anglian general practitioners were requested to search their prescription database for drugs used in PD. From this a list of probable patients with PD was generated and individuals were contacted by their general practitioner to ask if they were a twin. Zygosity was ascertained by questionnaire.

One hundred and fifty three surgeries responded $(47 \%)$ giving a study population size of about 1.3 million. Assuming the prevalence rate of $P D$ to be $1: 520,2500$ cases were expected; 2182 letters were sent to patients resulting in 1883 replies $(86 \%)$. Assuming a twin rate of $1: 40,54$ index twins were expected from the original sample, and 49 were found. None were doubly ascertained. Using stated zygosity, there were nine monozygous, 20 dizygous, and six stillborn twins and 14 of unknown status. The expected proportion of monozygous twins calculated by Weinberg's rule was 0.31 , which was exactly that found. These results were achieved in four months and the sample size already exceeds those of all but one published study.

The new method has provided a representative sample of twins with PD in rapid time. The method described could readily be applied to other neurological diseases, when patients are on long term medication. $[\mathrm{ABN}]$

GENERIC PRESCRIBING FOR EPILEPSY. IS IT SAFE?

P Crawford, B Chappell, J Collings, WW Hall, A Stewart. Bootham Park Hospital, York, UK

Previous generic prescribing studies in small groups of healthy volunteers or potentially biased groups of people with epilepsy have provided conflicting results. This study recruited people with epilepsy from a large variety of backgrounds in the community. Forty general practices in Yorkshire took part providing a patient base of over 350000 with 2285 being treated for epilepsy. All were contacted by their general practitioner and requested to complete a standard questionnaire. Those who had received a different supply of the same antiepileptic drug over the past two years were asked to report their experiences after the "switch". Those reporting adverse events were interviewed by their general practitioner. A full appraisal of the perceived problem was undertaken and only when no other explanation could be found was it concluded that the "switch" of antiepileptic supply was the causation of the problem.

A total of $1343(58.8 \%)$ responded; 251 $(18.7 \%)$ responders had experienced a "switch"; $74(29.5 \%)$ reported perceived problems, of which $27(36.5 \%)$ were validated by their general practitioner, 25 $(33.8 \%)$ were found to have other possible reasons for the perceived problems, and 22 $(29.7 \%)$ would not allow follow up; 177 $(70.5 \%)$ reported no problems.

Nearly a third of people after a "switch" reported perceived problems, of which 
$36.5 \%$ were validated. This study, therefore suggests that money saved by generic prescribing is outweighed by negative health gain for the person with epilepsy, increased work in general practice, and increased social costs.

[ABN]

SOME FASCICULATIONS IN MOTOR NEURON DISEASE HAVE A CENTRAL ORIGIN KR Mills. The Radcliffe Infirmary, Oxford, UK

Fasciculations, spontaneous discharges of motor unit potentials which are not under voluntary control, are generally thought to originate from peripheral nerve or axon terminals in muscle. In a study of the responses of single motor units to magnetic brain stimulation in motor neuron disease (MND) fasciculations have been detected which can be caused to fire by magnetic scalp stimuli. In 33 patients with MND, 22 had spontaneous fasciculations in the first dorsal interosseous muscle and in three patients it could be shown that the fasciculation potential could be activated by cortical stimulation, but was not among the first recruited motor units on voluntary action. These fasciculation potentials had simple waveforms by contrast with the complex waveforms of fasciculations originating in axon terminals. The three patients showing this phenomenon all had pure lower motoneuron signs, had low resting thresholds for cortical excitation, and had larger than normal excitatory responses. This suggests that cortical stimuli in these patients release large synchronous excitatory postsynaptic potentials capable of driving the motoneuron which is not capable of being driven by the relatively asynchronous input during volition. It is concluded that in MND, occasional fasciculations arise from sites central to the spinal motoneuron. [ABN]

REVERSIBILITY OF ISCHAEMIC DAMAGE DETERMINED BY MAGNETIC RESONANCE SPECTROSCOPY IN HUMANS

DE Saunders, FA Howe, A van den Boogaart, JR Griffiths, MM Brown. St George's Hospital Medical School, London, UK

The aim of this work was to study the ischaemic penumbra in humans, by measuring metabolic changes using proton magnetic resonance spectroscopy (' $\mathrm{H}-\mathrm{MRS}$ ).

Diagnostic MRI and ${ }^{1} \mathrm{H}-\mathrm{MRS}$ (TE $=30$ $\mathrm{ms}$ ) were performed on a $1.5 \mathrm{~T}$ system. ${ }^{1} \mathrm{H}$ MRS was carried out within the area of infarction. Peak area estimates were obtained using the variable projection time domain fitting analysis. The unsuppressed water signal was used as an internal standard. Two patients were studied within 17 hours of stroke onset. Both patients made a complete functional recovery.

The area of infarction was determined using T2 weighted images. $\mathrm{N}$-acetyl aspartate (NAA), a neuronal marker, and total creatine $(\mathrm{PCr} / \mathrm{Cr})$ were initially reduced in the region of infarction. In one patient, the NAA concentration partially recovered by week 2 at the time that the T2 weighted image returned to normal and the patient had made a complete recovery. The $\mathrm{PCr} / \mathrm{Cr}$ concentration partially recovered by week 1 and continued to recover up to week 3 . In the second patient, NAA and $\mathrm{PCr} / \mathrm{Cr}$ concentrations continued to fall up to one week post infarction. By week 2 a partial recovery of NAA and $\mathrm{PCr} / \mathrm{Cr}$ concentrations was made which continued for up to three months post infarction as the infarct size diminished on the T2 weighted images.

There was partial reversibility of NAA and $\mathrm{PCr} / \mathrm{Cr}$ in a region of infarction in two patients who have made a complete functional recovery after a stroke. This seems to demonstrate the metabolic recovery of neurons in the region of infarction. [ABN]

BRACHIAL ARTERY APPROACH CORONARY ANGIOGRAPHY: A CAUSE FOR HIGH MEDIAN NERVE PALSY?

AM Kennedy, M Grocott, $M$ Scott, $H$ Modarres, D Rowland, M Schwartz, F Schon. Atkinson Morley's Hospital, London and Queen Mary's Hospital, Roehampton, UK

Cardiac angiography (CA) is usually performed via the femoral artery approach When there is femoral artery disease or when aortic valve pressure monitoring is required, a right brachial artery approach is used. This study has identified five right handed patients who developed a high median nerve palsy (HMNP) after right brachial artery CA during the past three years. One patient had an extensive forearm haematoma which was confirmed on MR and required surgical exploration. Two cases were due to direct nerve trauma. One patient later developed causalgia which required treatment with sympathetic nerve blocks. The initial neurological deficits showed some improvement with time (follow up period six to 22 months). Nerve conduction studies confirmed the location of the deficit and showed some improvement with time. At follow up, all had significant impairment on the dexterity and stereognosis subcomponent of the Chessington hand assessment battery. This suggests a persistent significant disability in hand function. Peripheral nerve damage has been described after axillary and femoral angiography but HMNP has not been described as a complication of brachial artery CA. Haematoma formation must be excluded in patients with this complication. Direct nerve trauma and damage to the median nerve vascular supply are also contributory mechanisms.

[ABN]

DOES NOCTURNAL NON-INVASIVE POSITIVE PRESSURE VENTILATION REDUCE RESPIRATORY MUSCLE FATIGUE IN THE PALLIATION OF BREATHLESSNESS IN MOTOR NEURON DISEASE?

GH Mills, MI Polkey, CM Lloyd, D
Kyroussis, CH Hammegard, M Green, PN Leigh, J Moxham. Royal Brompton and King's College Hospitals and Institute of Psychiatry, London, UK

It has been postulated that resting the respiratory muscles may relieve fatigue and so improve respiratory muscle strength in ventilatory failure. However, there may be other reasons for an improvement in blood gases.

Two patients with motor neuron disease developed ventilatory failure with severe morning headaches, daytime drowsiness, and poor nocturnal sleep pattern. Respiratory muscle strength was assessed in both subjects. Nocturnal non-invasive positive pressure ventilation via a nasal mask (NIPPV) was commenced to assess whether these symptoms could be palliated. Respiratory muscle tests and blood gases were repeated after non-invasive ventilation to assess whether an improvement in respiratory muscle strength and arterial gase occurred. The results obtained are shown in the table.

Relief from the symptoms of ventilatory failure occurred very rapidly, and this was still the case four and six months after the introduction of NIPPV. Blood gases returned to normal, and remained normal even in the evening, when NIPPV had not been used all day. Respiratory muscle strength was not improved using NIPPV, nor was dynamic lung compliance.

These data suggest that relief of fatigue or reduction in atelectasis is not the mechanism for the persistent improvement in blood gases; a more likely explanation is the control each night of hypoventilation and the restoration of normal ventilatory control. [ABN]

QUANTITATIVE MRI DETECTS A RANGE OF HIPPOCAMPAL DISEASE IN TEMPORAL LOBE EPILEPSY

W Van Paesschen, A Connelly, CL Johnson, JS Duncan. Institute of Neurology, London, UK

The object was to define the range of hippocampal sclerosis (HS) using quantitative MRI in patients with intractable temporal lobe epilepsy (TLE)

Eighty one patients with intractable TLE ( $M=34, F=47$, median age 37 range 16-47 years) were scanned. Routine MRI was normal or showed HS. All had hippocampal T2 (HCT2) maps and hippocampal volumes corrected for intracranial volume.

For group 148 patients (59\%) had hippocampal volume ratio (HCVR) $<0.87$ (3 $\mathrm{SD}$ below mean control values). For group 2 33 patients $(41 \%)$ had a HCVR $\geqslant 0.87$.

In group 1,40 patients $(83 \%)$ had a unilateral abnormality (high HCT2, low hip-

\begin{tabular}{|c|c|c|c|c|c|c|c|c|c|c|c|}
\hline Case & & $\mathrm{SaO}_{2}$ & $p O_{2}$ & $p C \mathrm{O}_{2}$ & $p H$ & $B i c$ & $\begin{array}{l}T w \\
P d i^{*}\end{array}$ & $\begin{array}{l}\text { Sitting } \\
\text { vital } \\
\text { capacity } \\
\text { (d) }\end{array}$ & $\begin{array}{l}\text { Maximum } \\
\text { sniff } \\
\text { oeso- } \\
\text { phageal } \\
\text { pressure }\end{array}$ & $\begin{array}{l}\text { Maximum } \\
\text { expiratory } \\
\text { pressure }\end{array}$ & $\begin{array}{l}\text { Dynamic } \\
\text { com- } \\
\text { pliance } \\
\text { (l/cm } \\
\mathrm{H}_{2} \mathrm{O} \text { ) }\end{array}$ \\
\hline \multirow[t]{2}{*}{1} & Pre-NIPPV & 93 & $9 \cdot 1$ & $6 \cdot 5$ & $7 \cdot 426$ & 31.9 & 1 & $1 \cdot 1$ & 20 & 30 & 0.09 \\
\hline & Post-NIPPV & 96 & $10 \cdot 8$ & $5 \cdot 0$ & $7 \cdot 411$ & $23 \cdot 8$ & 1 & $1 \cdot 2$ & 23 & 31 & $0 \cdot 11$ \\
\hline \multirow[t]{2}{*}{2} & Pre-NIPPV & 93 & $9 \cdot 4$ & $8 \cdot 2$ & $7 \cdot 343$ & $32 \cdot 6$ & 2 & $1 \cdot 2$ & 25 & 42 & $0 \cdot 18$ \\
\hline & Post-NIPPV & 95 & $9 \cdot 7$ & $4 \cdot 4$ & $7 \cdot 479$ & $24 \cdot 4$ & 3 & $1 \cdot 3$ & 23 & 41 & $0 \cdot 17$ \\
\hline
\end{tabular}

* Twitch transdiaphragmatic pressure (Tw Pdi) utilises cervical magnetic stimulation of the phrenic nerve.

All arterial gases are measured in KiloPascals. 
pocampal volume, or both), seven (15\%) had an additional mild abnormality on the contralateral side, and one had normal HCT2s and volumes.

In group 2, eight patients (24\%) had bilateral HS, four $(12 \%)$ had a (mild) unilateral abnormality, and $21(64 \%)$ had normal hippocampal measurements.

A combination of quantitative $M R$ hippocampal measurements is necessary to demonstrate the range of unilateral and bilateral hippocampal disease found in most patients $(74 \%)$ with intractable TLE. Both visual inspection of routine MRI and quantitative hippocampal measurements were normal in an important subgroup (26\%). [ABN]

HEAIACHES IN CASLAITY

J Craig, V Patterson, O Dornan. Mater Hospital Trust, Belfast, UK

To find out what happens to patients with headaches who attend an accident and emergency (A and E) department consecutive patients attending the $\mathrm{A}$ and $\mathrm{E}$ department of the Mater Hospital, a district general hospital serving north Belfast, were studied in a six month period. All had headache as their main symptom. The patients were not managed differently from usual, and what happened to them was recorded from their charts. There were 167 attendances with headache accounting for $0.9 \%$ of all attendances. About one third were referred by their general practitioner, the rest being self referrals. Fifty patients were admitted and serious structural pathology was found in 18. Nine of these were transferred to a neurology ward. Thirty of the patients admitted had an incorrect initial diagnosis. Patients referred by their general practitioner were more likely to be admitted than self referrals. No patient who was sent home had developed structural disease at three month follow up. Headaches in casualty were managed safely in the present study but they could be managed more efficiently if fewer of the patients without serious disease were admitted. Screening by a neurologist of patients thought to need admission might result in fewer admissions and therefore a more efficient service. [ABN]

MELATONIN IN INSOMNIA

CM Ellis, JD Parkes. King's College School of Medicine and Dentistry and Institute of Psychiatry, London, UK

The action of the pineal hormone melatonin on sleep was determined in a group of insomniac patients. The study was double blind $\mathrm{v}$ placebo. Melatonin $(5 \mathrm{mg}$ ) or placebo was given-each for a consecutive seven day period to 15 subjects with severe long term psychophysiological insomnia - at 2000. These insomniac subjects, who also described daytime subwakefulness, had a mean estimated total sleep time of $5.6(1 \cdot 7)$ hours per 24 hours but no disturbance of circadian rhythmicity, with mean bed time $22.9(0.8)$ hours, wake time $07.2(0.8)$ hours. Previous benzodiazepine treatment was stopped 14 days before study.

Seven subjects reported "better sleep" on melatonin but bedtime, sleep onset time, estimated total sleep time and wake time, arousal frequency during sleep, and subjective rating of sleep quality were similar in pretreatment, melatonin, and placebo periods. There was a slight improvement in excessive daytime sleepiness while taking melatonin $(\mathrm{P}<0.01)$

It is concluded that melatonin is not an effective "hypnotic" compound but may be of value in the treatment of insomnia when this is accompanied by a major circadian disruption of the sleep-wake cycle. [ABN]

MAGNETIC RESONANCE SPECTROSCOPY STUDIES IN MOTOR NEURON DISIASI: CM Lloyd, G Lemmens, K Ray Chaudhuri, J Dawson, LH Goldstein, SC Williams, PN Leigh. Institute of Psychiatry and King's College School of Medicine and Dentistry, London, UK

The aim of this study was to investigate whether proton magnetic resonance spectroscopy (MRS) can be used to demonstrate cortical neuronal damage, as measured by a reduced N-acetylaspartate (NAA) to creatine $(\mathrm{Cr})$ ratio, in patients with motor neuron disease (MND).

Patients were selected with clinically probable or definite MND according to the El Escorial criteria, and compared with age and sex matched controls. Data were collected from the occipital, motor, and prefrontal cortex.

Reduced NAA/Cr ratios were found in the motor and prefrontal areas in the MND patients compared with the controls. For the prefrontal area this had a level of significance of $\mathrm{P}<0.025$ and for the motor area of $\mathrm{P}<0.02$.

$\mathrm{NAA} / \mathrm{Cr}$ as measured by MRS provides evidence of cortical neuronal loss in MND. This supports our previous experience with PET studies, using regional blood flow in conjunction with motor and cognitive activation and the ligand "C-flumazenil, which have shown cortical neuronal loss in the prefrontal area. This study adds further evidence to support the theory that neuronal loss in the prefrontal region is a consistent feature in the pathology of MND. [ABN]

GENERAL PRACTITIONERS: MANAGEMIINT OH CEREBROVASCUI AR DISIEASE

C Allen, SS Pollock. The Canterbury and Thanet Stroke Project, Kent, UK

Because most patients with stroke are admitted to hospital the general practitioners' role is often overlooked. The Canterbury and

Thanet Stroke Project therefore sought responses from 170 general practitioners in east Kent on: (1) The identification and management of transient ischaemic attacks (TIAs); (2) factors influencing acute stroke admission; (3) their use of community services for stroke patients after discharge.

Over $80 \%$ of general practitioners returned completed questionnaires. $40 \%$ found the diagnosis of TIAs difficult, and annual estimates of incidence were 10 times higher than expected (mean 6 range 1-30). There was, however, agreement on management with $75 \%$ of respondents recommending risk factor identification and antithrombotic treatment initially and $89 \%$ referring after further episodes. Ninety per cent often cited lack of available care at home and the need for nursing care as a frequent cause of admission but less than half felt the need to confirm or investigate that the diagnosis justified admission to hospital.

Half were satisfied with discharge arrangements and information whereas all thought it necessary to routinely visit appropriate patients although very few actually do; $90 \%$ would use the family support worker but less than a fifth commonly used the Stroke Information Centre or Dysphasic Support Services.

In conclusion TIAs are overdiagnosed and the lack of alternative models of care locally influences admission more than the need to investigate an increasingly treatable disease. General practitioners' perception of their role after discharge differs from the expectations of patients. These findings emphasise the need for increased general practitioner awareness of stroke and the value of neurovascular clinics. [ABN]

A TECHNIQLE I:OR THI: ASSISSSMENT' OI INT'ERCOSTAI. NERVE CONIDCTION

VP Misra, BD Youl, RS Howard. The National Hospital for Neurology and Neurosurgery, London, UK

Neurophysiological examination of respiratory function in patients with neuromuscular disease has so far been restricted to phrenic nerve conduction studies and to needle EMG examination of the diaphragm, intercostal muscles, and other accessory muscles of respiration. Intercostal nerve conduction is not generally assessed because the only described method yields variable results, is time consuming, and requires multiple recordings from surface electrodes placed over the rectus abdominus muscles Moreover, far field effects from surrounding muscles tend to complicate the interpretation of the tracings.

A reproducible and rapid technique enabling the assessment of intercostal nerve conduction is described. A concentric needle electrode is inserted into the eighth or ninth intercostal space just medial to the angle of the ribs. Intercostal muscle fibres are identified according to their pattern of activation in cooperative subjects, or by trial stimulation. The intercostal nerve is stimulated in the appropriate intercostal space at proximal and distal sites. A terminal latency to the onset of the evoked muscle action potential and motor nerve conduction velocity along a segment of the intercostal nerve can be consistently obtained and in some instances "F" wave latencies can be measured. This robust method is well suited to be performed on patients in the intensive care unit.

[ABN]

DIXAMPHFTAMINI: TRLATMENT IN THL

NARC(OLEPTIC SYNIOROML

SCR Williams, C Ellis, M Dahlitz, S Chen,

$R$ Howard, A Simmons, M Brammer, E Bullimore, JD Parkes. King's College School of Medicine and Dentistry and Institute of Psychiatry, London, UK

A retrospective self report study of dexamphetamine treatment was carried out on 124 
patients with the narcoleptic syndrome. Mean treatment period was 21 years, mean dexamphetamine dose $16 \mathrm{mg} / 24$ (range 5-60) hours. The propensity to excessive daytime sleepiness was determined by the Epworth sleepiness scale. Dexamphetamine reduced sleepiness by about $20 \%$. There was a minor reduction in the propensity to cataplexy. Less than $10 \%$ of subjects with the narcoleptic syndrome on treatment reported a daytime sleep propensity in the normal range. Long term treatment was associated with weight increase, not weight loss. Factors limiting drug response included physician prescription of suboptimal drug dosages, development of tolerance, and frequent side effects.

The mode of action of dexamphetamine was investigated by functional MRI using a visual and auditory activation paradigm in three normal subjects and two narcoleptic patients before and 90 minutes after giving dexamphetamine $(10 \mathrm{mg})$. Pretreatment activation levels were much lowe in narcoleptic patients than in normal subjects. After dexamphetamine, visual and auditory cortical activation was increased in the narcoleptic patients but not in controls.

[ABN]

TRANSCRANIAL SONOGRAPHY AND MAGNETIC RESONANCE ANGIOGRAPHY IN ACUTE STROKE AR Kenton, AR Moody, RJ Abbott. Leicester Royal Infirmary, Leicester, UK

Transcranial colour coded sonography (TCCS) and magnetic resonance angiography (MRA) are two non-invasive techniques which allow imaging of the cerebral vessels. Both have been employed in the assessment of 14 patients with acute stroke.

Patients (age range 48-84 years) were studied within 24 hours of stroke onset (range 4-23 hours). Two patients had cerebral haemorrhages, the remainder had infarctions. Findings from TCCS and MRA were classified as follows: normal, middle cerebral artery (MCA) M1 occlusion either proximal or distal, and MCA M2 occlusion. Flow was graded as normal, low (pulsed wave Doppler velocity $<20 \%$ of norma hemisphere; attenuated vessel on MRA), or absent (no signal on Doppler interrogation; absent vessel on MRA).

Four patients had normal TCCS findings; MRA was normal in three of these patients the fourth patient (intracerebral bleed) had low flow in the M2 portion of the MCA. Three patients had normal anatomy on TCCS, but low flow patterns using pulsed wave Doppler. MRA showed two of these patients to have attenuated MCAs with low flow; the other patient had an occlusion of an M2 MCA branch. Seven patients were classified as having MCA M1 occlusions (four proximal; three distal) on TCCS. This agreed with MRA findings in six patients; the seventh patient had a distal Ml MCA occlusion on MRA but a proximal occlusion on TCCS

In conclusion, TCCS and MRA findings were similar in this study group. However, MRA was able to identify the M2 portion of the MCA, which is technically difficult using TCCS. TCCS and MRA should be viewed as complementary techniques in the assessment of the patient with acute stroke.

[ABN]
STRATEGIES TO ISOLATE THE GENE CAUSING THE X LINKED DYSTONIA-PARKINSONISM SYNDROME

AH Németh, G Haberhausen, I Schmitt, A Köhler, U Peters, AP Monaco, U Müller. The Wellcome Trust Centre for Human Genetics, Oxford, UK and Instut für Humangenetik der Justus-Liebig-Universität Giessen, Germany

The aetiologies of both Parkinson's disease and dystonia are known to include genetic factors although few genes have been isolated and analysed. As part of a long term study aimed at identifying genes which cause these disorders the $\mathrm{X}$ linked dystoniaparkinsonism syndrome which is endemic on the island of Panay in the Phillipines has been analysed. Clinically, there is severe generalised dystonia and parkinsonism which does not respond to conventional medication, and death ensues within 16 years of onset. The neuropathological features include neuronal loss and a mosaic pattern of astrocytosis in the caudate and lateral putamen. The gene causing this disorder (known as DYT3) is genetically homogeneous, which has facilitated fine mapping by linkage disequilibrium studies This has disclosed the location of DYT3 within a $1.8 \mathrm{Mb}$ region on the proximal long arm of the $\mathrm{X}$ chromosome (Xq13.1). A yeast artificial chromosome (YAC) contig has been constructed across the critical region and the YACs are being used to isolate candidate genes by a variety of techniques including screening for trinucleotide repeats, analysis of $\mathrm{CpG}$ islands, and exon trapping. The identification and characterisation of the DYT3 gene will provide important insights into the molecular basis of Parkinson's disease and dystonia. [ABN]

QUADRICEPS STRENGTH MEASUREMENT IN CLINICAL PRACTICE

MI Polkey, GH Mills, D Kyroussis, PN Leigh, M Green, J Moxham. King's College and Royal Brompton Hospitals and Institute of Psychiatry, London, UK

A supramaximal isometric quadriceps twitch may be obtained in normal subjects by magnetic stimulation of the femoral nerve and the twitch:MVC ratio (mean $0 \cdot 18$, range $0 \cdot 15-0 \cdot 23$ ) is comparable with the twitch: tetanus ratio previously reported in isolated skeletal muscle (Polkey et al. Clin Sci 1995;89:16P). Twenty patients with suspected muscle weakness have now been studied using a $70 \mathrm{~mm}$ double circular coil powered by a Magstim 200 DEM and the twitch tension (TwQ), surface EMG, and maximum voluntary contraction force (MVC) were measured. Care was taken to avoid unwanted twitch potentiation. Supramaximal stimulation was obtained in all subjects as judged by plateauing of TwQ and EMG. The mean within occasion coefficien of variation was $5 \%$ (range $1 \cdot 7-12 \cdot 8 \%$ ). When judged by MVC 15 patients were weak (normal $>40 \mathrm{~kg}$ ) whereas by TwQ only 12 were weak (normal $>7 \mathrm{~kg}$ ). The mean TwQ:MVC ratio was $0 \cdot 26$ (range $0 \cdot 17-0 \cdot 43$ ). This suggests that some patients do not perform a fully maximal MVC. It is concluded that measurement of TwQ is a reproducible test of quadriceps strength which has particular advantages if the patient cannot properly perform an MVC manoeuvre.

[ABN]
OUTREACH AND HOSPITAL HEADACHE SERVICE

AJ Fowle, CG Clough. Kings College Hospital, London, UK

The aim of the Brook Headache Initiative is an improvement in the management of headache within the primary health care setting. A secondary aim is to improve access to hospital clinics when necessary.

The service employs a full time research registrar (AJF) holding one hospital clinic and three or four outreach clinics. General practitioners can refer patients in either setting. Management of patients is conventional, with access to investigation and general neurological advice. A range of leaflets is available for both patients and general practitioners.

One hundred and seventy nine patients were seen in the first six months, 70 in outreach clinics, 105 in the hospital clinic, and four in the general neurology clinic. Initially, hospital patients were taken from the neurology waiting list. As a result, the waiting time dropped from 78 weeks to 18 weeks As the waiting time fell, the non-attendance rate dropped from $50 \%$ to $0 \%$. Outreach clinics were also used as an opportunity to update general practitioners on the management of headaches.

Many patients had more than one headache. Migraine (38\% of diagnoses) and tension headache $(29 \%)$ were the most common diagnoses.

$[\mathrm{ABN}]$

PRESLEEP BEHAVIOU

G Lemmens, CM Ellis, JD Parkes. King's College School of Medicine and Dentistry and Institute of Psychiatry, London, UK

Many animal species wind down in preparation for sleep. Cats look around for a sleeping place, animals with bushy tails curl up with the tail over the eyes, and trunk movements slow down in the elephant.

The behaviour of 90 normal subjects aged 17-79 (mean, 49) years, 37 men, 53 women, in the two hour presleep period was studied on a midweek winter evening.

In the two hours before sleep, one third of all subjects took some exercise, $23 \%$ of subjects showed checking behaviour of door and window locks. Sixty per cent set an alarm clock, and $24 \%$ prepared clothes for the following morning. Half of all subjects had a drink, snack, or meal. All subjects went to the bathroom before going to bed and spent a mean 9 (6) minutes there Twenty two per cent of subjects had a bath or shower. Mean sleep preparatory latency, from the time of first preparation to sleep onset, was 77 (48) minutes. The mean estimated bedtime was $23.29(0.93)$ hours with lights out at $23.56(0.87)$ hours and sleep onset time, $24.00(0.97)$ hours.

Both learned and unconditioned mechanisms are important in human presleep behaviour.

$[\mathrm{ABN}]$

SELECTIVE IMPAIRMENT FOR SIMPLE DIVISION L Cipolotti, A Costello. Brook General Hospital and National Hospital fo Neurology and Neurosurgery, London, UK 
A patient with a calculation impairment is reported. He had a very good premorbid knowledge of calculation, including division problems. After a series of vascular events he presented with a severe deficit in solving simple division problems, such as $4 \div 2=$ ?

He was also found to be impaired in solving simple arithmetical problems such as $3 \times$ ? = 6 . By contrast his performance on simple and complex addition and subtraction problems was relatively well preserved. Remarkably, his ability to solve simple and complex multiplication problems, even those requiring carrying (for example, $95 \times 76$ ) was also well maintained. Such a clear cut dissociation has not been previously documented. The implication of these findings for the organisation of stored arithmetical facts are discussed.

[BNS]

COGNITIVE PROCESSES INVOLVED IN THE APPRECIATION OF NEW METAPHORS R Corcoran. University College, London, UK

A recent PET study has shown that the appreciation of new metaphors activates a distributed cognitive system considered to be related to the retrieval of personal episodic memory. Areas of medial parietal and right prefrontal cortex are among those specifically activated during a test in which the subject must decide whether or not an unfamiliar metaphor is plausible or not. This presentation provides further evidence in support of this notion by examining the performance of three contrasting case studies on the metaphorical appreciation task and a literal sentence control task. Two cases of Korsakoff syndrome present very similar neuropsychological profiles but remarkably different abilities on these tests. The third case is that of a thought disordered schizophrenic whose performance enlightens neuropsychiatric understanding of these patients. The cases demonstrate how subtle differences in episodic memory impairment in harness with deficits in particular executive skills, the purpose of which is to enable meaningful communication, can lead to such contrasting performance not only on these new tests but also in day to day social functioning. [BNS]
SEVERE AMNESIA AND HIPPOCAMPAL NEURON LOSS: A CASE OF UNUSUAL LATE

COMPLICATION AFTER TEMPORAL

LOBECTOMY WITH NECROPSY FINDINGS

SM Oxbury, MV Squier, S Renowden, KN

Carpenter, JM Oxbury. Radcliffe Infirmary, Oxford, UK

Severe amnesia, a rare immediate consequence of temporal lobe epilepsy surgery, is seldom, if ever, reported as a late complication. The medial temporal lobe area critical for severe amnesia is poorly understood.

A patient underwent uncomplicated en bloc left temporal lobectomy $(6 \mathrm{~cm}, 43.5$ gm, hippocampal sclerosis) aged $19 . \mathrm{He}$ remained seizure free for eight years until a convulsion followed an accidental head injury. $\mathrm{He}$ became severely amnesic after a fourth convulsion 16 months later.

$\mathrm{He}$ was right handed; preoperative IQ average, verbal memory poor, non-verbal memory normal. Five years postoperatively these were unchanged. After the first postoperative seizure he worked successfully and began a professional training. After onset of amnesia his IQ was unchanged; anterograde memory severely reduced; no delayed recall of new material; retrograde amnesia dense for 16 months between first postoperative seizure and amnesia onset. Very slight improvement occurred. He died two years later.

MRI before amnesia showed absence of anterior left temporal lobe, preservation of superior temporal gyrus, atrophy of left fornix and mamillary body, normal right temporal lobe. Four months after onset of amnesia right hippocampal volume had reduced by $35 \%$.

Necropsy showed: previous left temporal lobectomy; atrophy of left fornix and mamillary body; neuronal loss in right hippocampus, severe in CA1, CA4; intact right amygdala; recent diffuse damage associated with cause of death.

In conclusion, a convulsion can cause severe hippocampal damage in adult life; the patient was left with bilateral loss producing amnesia; necropsy suggests that critical zones are CA1 and/or CA4; the amygdala alone cannot support acquisition of new material.

[BNS]
COGNITION AND MULTIPLE SCLEROSIS: A HISTORICAL ANALYSIS OF MEDICAL

PERCEPTIONS

J Richardson, A Robinson, I Robinson. Department of Human Sciences, Brunel University, London, UK

This paper is a study of accounts of cognitive disorders in multiple sclerosis (MS) from the time of the earliest reported cases in the mid-19th century. It demonstrates that cognitive disorders were only relatively rarely distinguished from the general category "mental symptoms" in relation to which a broad range of affective disorders was often considered a crucial indicator. Case study methods led to many disputes about the extent and nature of such symptoms, exacerbated by different national traditions in England, Germany, France, and the United States.

What are now recognisably appropriate scientific methods were only used in a modest number of studies in the 1950s and the $1960 \mathrm{~s}$ in relation to cognitive issues, and it was still argued as late as the mid-1970s that affective rather than cognitive processes were the key to understanding the psychological aspects of MS. Major changes in the early 1980s in the development of methodologies for the detection of subtle and widespread cognitive changes in MS; in the use of MRI techniques to study further the link between cognitive deficits and other pathological processes, and in the increasing collaboration between neurologists and neuropsychologists, accelerated work in this area.

In conclusion, the development of research in the cognitive aspects of MS has not been a steady and linear process, but one that has been hesitant and problematic. Paradoxically recent interest in these processes has disclosed their complexity, has led to considerable debate about methods, and has focused attention on the need for further research. In addition, the pressure to use the findings of such studies to improve patients' everyday lives, has indicated the necessity to consider again the possible association between affective and cognitive processes-a link which much 20th century neuropsychological research has been concerned to undermine.

[BNS] 